Preprint version of accepted manuscript, to be published in the Journal of Paleontological Techniques

\title{
Photogrammetry in ichnology: 3D model generation, visualisation, and data extraction
}

Jens N. Lallensack ${ }^{1,2 *}$, Michael Buchwitz ${ }^{3}$, and Anthony Romilio ${ }^{4}$

${ }^{1}$ Section Paleontology, Institute of Geosciences, Rheinische Friedrich-Wilhelms-Universität Bonn, Bonn, Germany

${ }^{2}$ School of Natural Sciences and Psychology, Liverpool John Moores University, James Parsons Building, Bryon Street, Liverpool L3 3AF, UK

${ }^{3}$ Museum für Naturkunde Magdeburg, Otto-von-Guericke-Str. 68-73, 39104 Magdeburg, Germany

${ }^{4}$ School of Chemistry and Molecular Biosciences, The University of Queensland, Brisbane, Qld 4072, Australia

*corresponding author jens.lallensack@gmail.com 


\begin{abstract}
3D digitisation of surfaces became a standard procedure in ichnology in recent years. 3D models allow not only for the digital preservation of vulnerable ichnological records, but also for the illustration, qualitative description, and quantitative analysis of the fossils. Here we discuss how to obtain photographs for photogrammetry, to generate, scale, and orient the models, and to extract visualisations, measurements, and coordinates for data analysis. Different visualisation approaches are discussed and compared, including widely used techniques such as orthophotos, height maps, and contour maps, as well as rarely used yet promising methods such as low-angled shaded reliefs, surface inclination plots, ambient occlusion, and radiance scaling. These techniques may filter or enhance different properties of the model surface such as colour information, elevation, edges and slopes, 3D morphology, and specific features such as convexities or concavities, and will often reveal additional detail. Furthermore, an approach is presented to automatically calculate trackway parameters based on coordinates collected from the model visualisations. All discussed steps can be performed with free and open-source software, and we provide detailed software instructions. We argue that digital ichnology, when combined with sedimentological data of the site, can be equally or more effective and comprehensive as traditional ichnological fieldwork.
\end{abstract}

\title{
INTRODUCTION
}

The use of 3D digitisation techniques in ichnology has proliferated during the last decade, and now is widely considered a standard procedure in the field (Falkingham et al., 2018). The benefits of these new set of methods can not be underestimated, and include the proper collection, dissemination, and long-term archiving of data that can be visualised and analysed both qualitatively and quantitatively. Photogrammetry, which allows to calculate 3D-models from photographs, became the most frequently used 3D digitisation method in ichnology (Matthews and Breithaupt, 2001; Falkingham, 2012; Falkingham et al., 2018). Using simple photographs as data source, it is applicable at low costs and allows for capturing the $3 \mathrm{D}$ morphology, including photo-realistic texture in high resolution. 
We here argue that 3D models of tracks and tracksites can be as effectively and comprehensively analysed as any traditional fieldwork would be able to, provided that they get combined with sedimentological data of the tracksite. The potential of 3D models in the description, illustration, and analysis of tracks goes beyond what was possible with traditional methods, and these new possibilities have not yet been fully explored and remain underused. Interpretive outline tracings are inherently subjective and do not capture the 3D morphology (Falkingham, 2016), while single photographs can be difficult to interpret even with optimal light conditions (Thulborn, 1990) and introduce perspective distortion. Such shortcomings can be easily overcome with 3D models, which can be projected two-dimensionally in orthographic view (without perspective distortion) and rendered using a broad array of techniques that can filter or emphasize properties such as colour information, elevation, edges and slopes, 3D morphology, or specific features such as convexities and concavities. These methods frequently allow for recognizing additional footprints or shape features that are not easily recognisable in the field, especially when lighting is not optimal. The different projections can be used to digitally interpret and measure the features of interest, and to draw interpretative drawings and site maps.

In the present paper, we discuss producing, visualising, and analysing 3D data of traces and tracksites. The process involves taking photo series of the fossil or site, model generation, model post-processing, visualisation, and data extraction, and is summarised in Fig. 1. We will discuss the most common mistakes in photographing tracks for photogrammetry, and outline how to generate, scale, and orient the 3D models. We then evaluate and compare different techniques for the visualisation of ichnological 3D data, providing recommendations for the choice of methods and parameters for different types of structures, including high-relief and low-relief surfaces as well as skin impressions. We continue with discussing the extraction of data including measurements, outline drawings, sitemaps, and coordinates. Finally, we will present a method to automatically calculate trackway parameters based on coordinates taken from the models.

Multiple software solutions exist for each of the discussed in-software steps. For the purpose of accessibility, we will focus on a selected combination of software, for which we provide detailed instructions for the in-software workflow in the appendix of this paper. This software is free and open-source (FOSS). For the generation of 3D models we introduce Meshroom v. 
2019.2 (AliceVision, 2018). Model post-processing and visualisation will be achieved with MeshLab v. 2020.1 (Cignoni et al., 2008), CloudCompare v. 2.10 (CloudCompare, 2019), and ParaView v. 5.8.0 (Ayachit, 2019). Discussed methods for data extraction and analysis are based on the vector image editor Inkscape 1.0 (Inkscape, 2020) and the spreadsheet application LibreOffice Calc 6.2 (The Document Foundation, 2019). In addition, we briefly discuss the proprietary software Agisoft Metashape 1.6.0 Standard and Professional editions (known as Agisoft Photoscan before 2019; Agisoft, 2020), as this is the most widely used software for 3D model generation used in the field (AliceVision, 2018). All software discussed herein runs on various platforms including Linux, Windows, and, which the exception of Meshroom, MacOS.

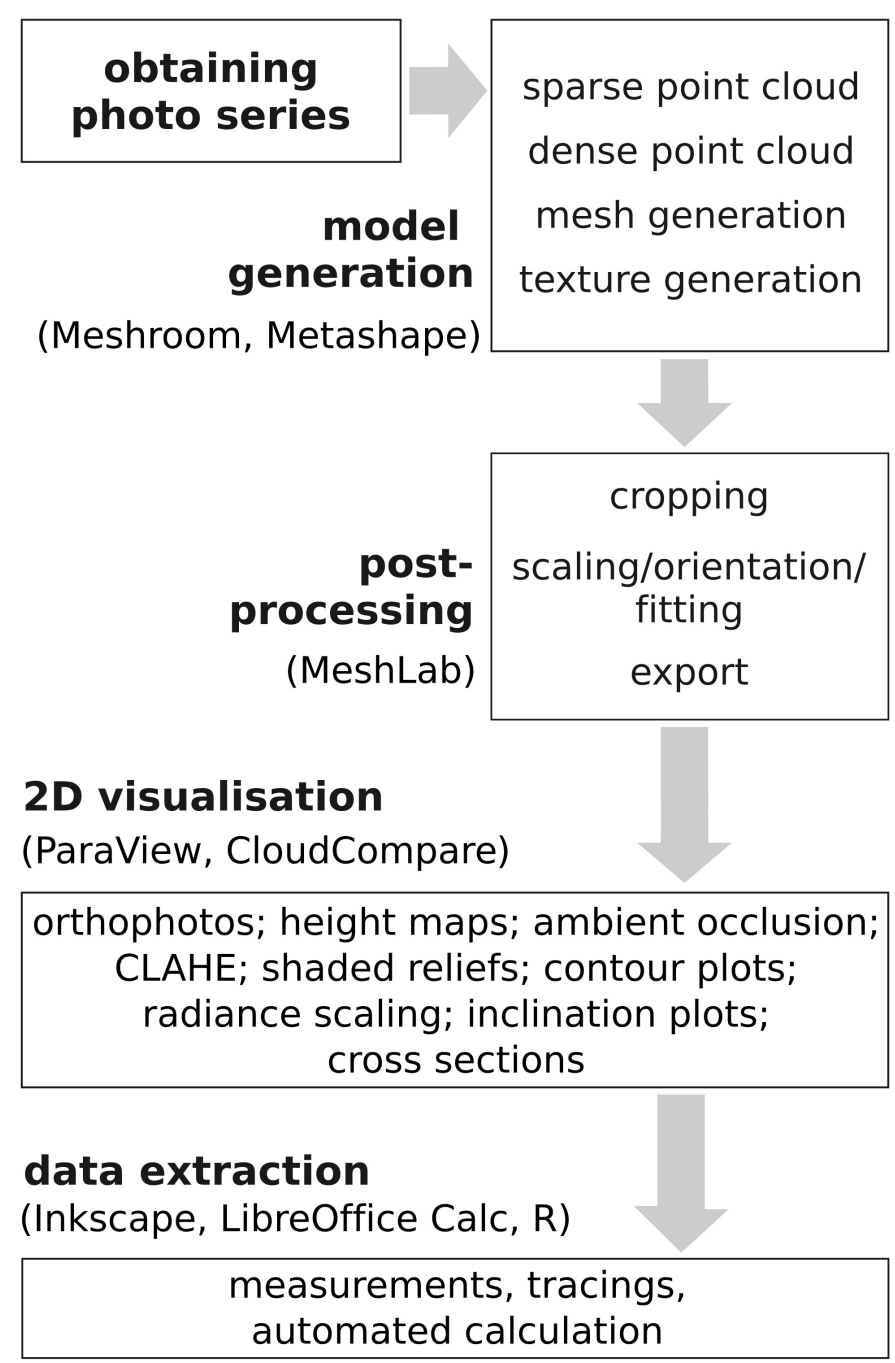

Fig. 1: Diagram showing the suggested workflow including obtaining images for photogrammetry, model generation (using Meshroom or Metashape), model post-processing 
(using MeshLab), 2D visualisation of the obtained 3D data (using ParaView and CloudCompare), and data extraction (using Inkscape, LibreOffice Calc, and R).

Although we aim to cover the most frequently used methods in digital ichnology, the set of discussed methods is not exhaustive. Important areas that cannot be covered here include the integration of photogrammetry, LiDAR, and geographic information systems (GIS) (Breithaupt et al., 2004; Matthews et al., 2006, 2016; Bates et al., 2008). The use of archival photographs to retrospectively reconstruct lost tracksites is discussed in Falkingham et al. (2014) and Lallensack et al. (2015). Belvedere (2018) introduced the concept of stat-tracks and mediotypes, the mean or median 3D morphology of a set of footprints, to allow for comparisons of morphology while suppressing features unrelated to trackmaker anatomy. Methods for the production of shape difference maps, which can highlight erosion of a site over time or differences between the mould and cast of a single footprint, are described in Matthews (2008) and Wings et al. (2016), respectively.

\section{TAKING PHOTOGRAPHS FOR PHOTOGRAMMETRY: COMMON MISTAKES}

Photographs for photogrammetry of traces and tracksites should adequately cover the whole object and have sufficient overlap, be in-focus, taken perpendicularly above the surface, and taken at different camera positions at each shot. Scale bars need to be placed with the object; shadows avoided; and surfaces cleaned.

In the following, we list the - from our experience - most common pitfalls in acquiring quality photographs for photogrammetry of ichnological fossils and sites. More extensive discussions on photographing and model generation can be found elsewhere, for fossil footprints (Matthews, 2008; Matthews, Noble \& Breithaupt, 2016) and body fossils (Mallison \& Wings, 2014). Camera choice is discussed in Matthews (2008) and Mallison \& Wings (2014).

- Insufficient photograph coverage. Photographs should optimally have $2 / 3$ overlap with each other if taken at a constant distance to the object. Tracksites are best photographed systematically, to ensure that the complete surface is covered and 
sufficient overlap is present in all cases. Photographs at very close distances, especially when using a macro objective, may require increased overlap if the depth of field does not bring all areas captured by a photo into focus. Regions which are only visible close to the margins of the photographs may show reduced quality in the final model; it is therefore important that all areas of interest, especially those close to the edges of the model, are captured at the centres of photographs.

- Blurry pictures. Modelling requires photographs to be well-focused and without motion and depth blur. For best image quality, the use of a tripod or camera stabilizer is recommended, and ISO should be set to a low value in the camera settings to minimize noise. Hand-held photographs may lead to sufficient results when enough light is present and the object is not too close to the camera. Moderately higher ISO values may be necessary when taking hand-held photographs under less favourable conditions to reduce motion blur. When taking hand-held photographs, we recommend taking three independent sets of photographs, especially when the tracksite cannot be revisited easily, so blurry pictures can be replaced. When photographing with autofocus enabled, the metering mode should be set to "Centre" and the focus updated at every shot (see below for photographing with manual focus). When photographing small objects, and especially in Macro photography, the use of a tripod and mid to high f-numbers for a large depth of field is crucial. Small objects are often easier to capture with a small camera sensor (e.g., phone cameras) due to the higher depth-of-field. The depth-of-field may also be increased by using focus stacking (Santella \& Milner, 2017). We recommend exclusion of any blurry pictures before model generation.

- Oblique photographs. Vertical shots are optimal as long as overhanging parts are absent and the track walls are less than vertical. Low-angled photographs need to be avoided. Very small traces, especially when using macro photography, require both vertical and slightly angled (15-20 from the vertical) photographs for optimal alignment.

- Singular camera position/pivot. The position of the camera needs to be changed with each shot. Avoid making photographs by pivoting the camera at a fixed position that otherwise results in a 'fish-eye effect'. 
- Suboptimal distance between camera and object. The resolution (and file size) of the model will primarily depend on the distance of the camera from the object. At closer distances, however, determining sufficient overlap becomes more difficult in practice because of the higher number of photographs required. Model resolution may be locally increased by taking pairs of photographs with reduced distance, to capture fine detail such as skin impressions. Changes in distance should not be lower than $50 \%$ (when taking close-ups) or higher than $200 \%$ (when taking overview shots) to ascertain continuity between shots (Matthews et al., 2016).

- Lack of scale. As photogrammetry is scale-independent, the scale of the model needs to be set manually. For this reason, including one or more scale bars within the model is crucial. These scale bars should be large and well-readable in the centre of several photographs, respectively, but in a position that does not obscure important target objects.

- Shadows. Surfaces are ideally photographed at times when no sharp shadows occur, e.g. when overcast or at noon, as a proper and even exposure of images is crucial. Varying lighting conditions and moving shadows need to be avoided. When photographs of a set are to be taken at different dates, it needs to be ascertained that the sun angle is consistent (Matthews et al., 2016). Although using compressed image formats such as JPEG are sufficient in normal circumstances when set at maximum quality (Mallison and Wings, 2014), RAW image formats allow for exposure adjustment during post-processing, potentially providing more detail in over- or underexposed areas (Matthews, 2008).

- Unclean surfaces. Tracksites should be cleaned, and any water and covering vegetation removed, before photographing (Matthews, 2008).

- Automatic rotation and cropping. Automatic image rotation needs to be turned off in the camera settings (Matthews, 2008). It is important not to crop any images.

- Insufficient planning and lack of equipment. Depending on the specifics of the site, required equipment may include backup batteries and memory cards; tripods of varying heights, with extension arms that can be mounted horizontally for shooting down; camera stabilizers; scales; tape (for scale fixation); sponges (for water removal); 
cleaning utilities; strings (for guidance on a larger surface); monopods and ladders (for inclined surfaces); camera remote controls; plastic sheets (to diffuse sunlight), GPS units, and drones (Matthews, 2008; Mallison and Wings, 2014; Matthews et al., 2016). It is practical to start a series with overview shots of the tracks or tracksite for context, followed by at least three photographs that show each scale bar close to the centre of the image and in full focus.

For highest accuracy, we recommend maintaining a constant distance between camera and object, and a constant zoom and focal length, especially when shooting vertical photographs (Matthews, 2008; Matthews et al., 2016). A constant focal length can be achieved by using manual focus; increased care should be taken that all photographs are in focus. The automatic camera calibration of the photogrammetry software can be improved by adding two additional and redundant photographs with the camera rotated $90^{\circ}$ and two with the camera rotated $270^{\circ}$ (for details see Matthews, 2008; Matthews et al., 2016).

\section{MODEL GENERATION}

Model generation can be done in four principal steps: camera alignment and sparse point cloud generation; dense cloud generation; mesh generation; and texture generation. During the first step, the camera position is calculated for each photograph based on features (pixel combinations) that could be matched between photographs. These features, or tie points, will likewise be projected into 3D space and form the sparse point cloud, which may already serve as a preview of the final model. As the second step, the dense cloud generation, is the most time consuming, it is advisable to check the sparse cloud and the aligned cameras (the calculated camera positions, which can be displayed around the sparse cloud) for obvious errors before proceeding. The meshing step reduces the dense cloud to a single surface of connected polygons. Although the dense cloud can be used for research purposes, some visualisation approaches require a meshed model (i.e., those involving shading with virtual light). The fourth step, the texture generation, is optional for including photorealistic colour information (see section "Colour information"). 
Although several software solutions for photogrammetry exist, we restrict ourselves to briefly introduce two solutions, Meshroom and Agisoft Metashape, which both cover the entire process of obtaining models from images. Meshroom is free software and easy to use but requires a CUDA enabled GPU (i.e., a Nvidia graphics card). The alternative, Metashape, is proprietary software and runs on a broader range of hardware. As photogrammetry software is currently still developed at a fast pace and workflows may change from one version to another, we refrain from providing detailed instructions on the model generation process itself, but refer the reader to the user manuals of Meshroom and Metashape. However, we do provide short instructions to get started with the current versions of both packages, and offer some advice on in-program settings specific for ichnological fossils, in the Appendix of this paper.

The quality of the resulting photogrammetric model should be assessed prior to its further use. Virtual inspections provide a first quality check, including comparisons with photographs (Carbonneau et al., 2003). Noise, such as small-scale spikes leading to a roughened surface, best seen under low-angled virtual light, or artefacts such as linear or concentric wave-like structures are a typical indicator of more severe error and might be confused with original features (Lallensack et al., 2015, fig. 5). A quantitative measure of overall model quality is the root-mean-square error (see discussion in Matthews, 2008). It should be noted, however, that quality may differ drastically in separate regions of a model and decreases towards the model edges. Metashape allows for filtering tie points based on their estimated errors, which may improve model quality (see Appendix for details).

\section{MODEL POST-PROCESSING}

The generated model requires post-processing in other software prior to $2 \mathrm{D}$ visualisations of the 3D model surface, including cropping, scaling, and orienting. Models may include colour information, which can be optimised after model generation. We here point to the respective functions in MeshLab (see Appendix for detailed instructions), but note that most steps can be alternatively achieved in CloudCompare or Metashape. 


\section{Cropping}

Noisy surroundings of models and slab edges or other regions that are not of interest should be cropped away. This can reduce model relief and facilitate the generation of graphical output. Various cropping tools are available in MeshLab, including the "Select Faces in a rectangular region" tool and the more flexible "Select Faces/Vertices inside polyline area" tool. Both tools can select the area of interest and invert the selection, after which all surrounding areas can be deleted. Models may contain smaller parts disconnected from the main object, which can be automatically selected and deleted, e.g. in MeshLab using the "Remove isolated pieces (wrt Diameter)" filter.

\section{Scaling}

Photogrammetry is scale-independent, meaning that the size of the model has to be set to allow for measurements. Defining the scale of the model can be done in MeshLab, provided that at least one object of known length is present within the model. First, this object (e.g., a scale bar) is to be measured using the "Measuring" tool. This measurement is used to calculate the scale factor (actual length/length given by the "Measuring" tool). The model can then be scaled according to this factor using the "Transform: Scale, Normalize" filter. Accuracy can be enhanced, and error quantified, by calculating several scale factors based on additional scale bars, preferably placed on opposite sides of the object, and taking the mean of these scale factors.

Metashape Professional offers marker-based scaling, where at least two marker points are placed on the object of known length directly in the input photographs. This method is generally more accurate than defining the scale on the model itself. Markers for scaling may be detected automatically when using coded targets. 


\section{Model orientation}

2D visualisations of tracks should be in precise top view, allowing shape comparisons and measurements, although additional inclusion of oblique views can be helpful to improve depth perception (Falkingham et al., 2018). Where overhangs obscure track morphology, the model can also be shown in bottom view by switching the z-axis.

To achieve a precise model orientation, the plane spanned by two of the three axes (the $\mathrm{x}$ and $y$-axes) should be identical to the surface plane of the model (henceforth termed the horizontal plane). Manual adjustment of the model to the desired orientation is inaccurate and can be avoided. Automatic fitting to the horizontal (xy) plane can be easily done in MeshLab using the "Transform: Rotate to fit to a plane" filter (the alternative "Transform: Align to Principal Axes" filter should be avoided as it can lead to mirroring of the model). Before applying the filter, the model needs to be selected. It is possible to select only parts of the model that will then serve as a reference for the horizontal plane to exclude areas of high relief from the fitting process. Per default, the filter will also centre the model on the origin of the coordinate system. After fitting to the horizontal plane, the model can be rotated around the z-axis into the desired orientation (e.g., to have digit impressions facing upwards) using the "Transform: Rotate" function. Model orientation can be checked by examining the orientation axes (the "Show Axis" function of MeshLab). If the "Front" view displays the underside of the model rather than the upper side, the model needs to be rotated around the $x$ or $y$-axis by $180^{\circ}$.

\section{File export and textures}

The most commonly used formats to distribute photogrammetric 3D data include the Stanford Polygon File Format (ply) and Wafefront OBJ (.obj). Both formats support the inclusion of colour information (see below).

An important advantage of photogrammetric models over other 3D digitisation methods is the inclusion of colour information. A single colour value can be associated with each vertex of the 
model. When using the OBJ or PLY formats, these vertex colours can be directly stored within the model. With vertex colours, however, the resolution of the colour information is limited to the resolution of the mesh. Much higher resolutions can be achieved by calculating a photorealistic texture, which can be done with both Meshroom and Metashape. When exporting with texture included, the model file will be accompanied by one or more additional texture files (usually JPEG). When exporting to the obj format, there will be a $\mathrm{mtl}$ file in addition to the model and texture files. Textures can be used instead of or in addition to vertex colours. To ensure that different software solutions can load the texture, the model file name should avoid spaces.

\section{D VISUALISATION}

3D data of trace fossils can be visualised using a variety of methods, including orthophotos; shaded reliefs; height maps; contour plots; ambient occlusion; radiance scaling; and crosssections. In the following, we introduce each of these methods and discuss their utility for enhancing different features of interest. For the generation of the different 2D visualisations, we recommend three free software solutions: MeshLab, ParaView, and CloudCompare. MeshLab is well-suited as a quick and performant viewer of 3D models, while ParaView and CloudCompare allow for a broader array of graphical outputs. All three software solutions allow for exporting high-resolution visualisations. Detailed instructions for the creation of these graphical outputs are provided in the Appendix. Respective options in Metashape Professional are mentioned when present; we refer the reader to the Metashape Manual (Agisoft 2020b) for detailed instructions.

\section{Perspective vs. Orthographic view}

Per default, the discussed 3D software will correct for perspective, so that any screenshots taken from the model are affected by perspective distortion - as is the case with photographs. Alternatively, 3D models can be displayed in orthographic projection, where the projection 
lines are parallel to each other. Orthographic projections are projected as maps, i.e., the scale is uniform across the image. We recommend using orthographic projections, especially when the depicted track is shown in top view. Orthographic projections allow for precise comparisons of separate models and allow for taking measurements directly from these 2Drepresentations. The discussed 3D software allows for toggling between perspective and orthographic mode. In ParaView, the equivalent option is termed "Camera parallel projection".

\section{Colour information and CLAHE}

Increasing contrast often makes additional detail visible. Vertex colours of a model can be adjusted for contrast, brightness and gamma in MeshLab, while separate texture files and 2D visualisations can be directly manipulated using standard image editors such as GIMP (www.gimp.org). However, contrast increase, whether manually or automatically using histogram equalisation, often results in over- or underexposure of separate parts of the image. This problem can be avoided using Contrast limited adaptive histogram equalisation (CLAHE, see Zuiderveld, 1994), which calculates separate histograms for different sections of the image, therefore achieving uniform contrast and brightness even when the source image is unevenly exposed to light (Fig. 2).

CLAHE can be applied to standard photographs (Fig. 2), texture files, and 2D visualisations such as orthophotos (Fig. 3A). It may also enhance detail in height maps, especially when features of low relief (e.g., individual tracks of a tracksite or skin impressions) are distributed over an uneven surface (Fig. 4C). It has to be noted, however, that absolute elevation cannot be interpreted from such modified height maps anymore. A plugin for the free image processing program ImageJ (imagej.net) is available (Saalfeld, 2009; see https://imagej.net/Enhance_Local_Contrast_(CLAHE)). Important parameters include the "block size", the size of the separate image sections to be adjusted, and the "max slope", which defines the intensity of the adjustment. The effect of the CLAHE plugin can be lessened by increasing "block size" and reducing "max slope", respectively. 

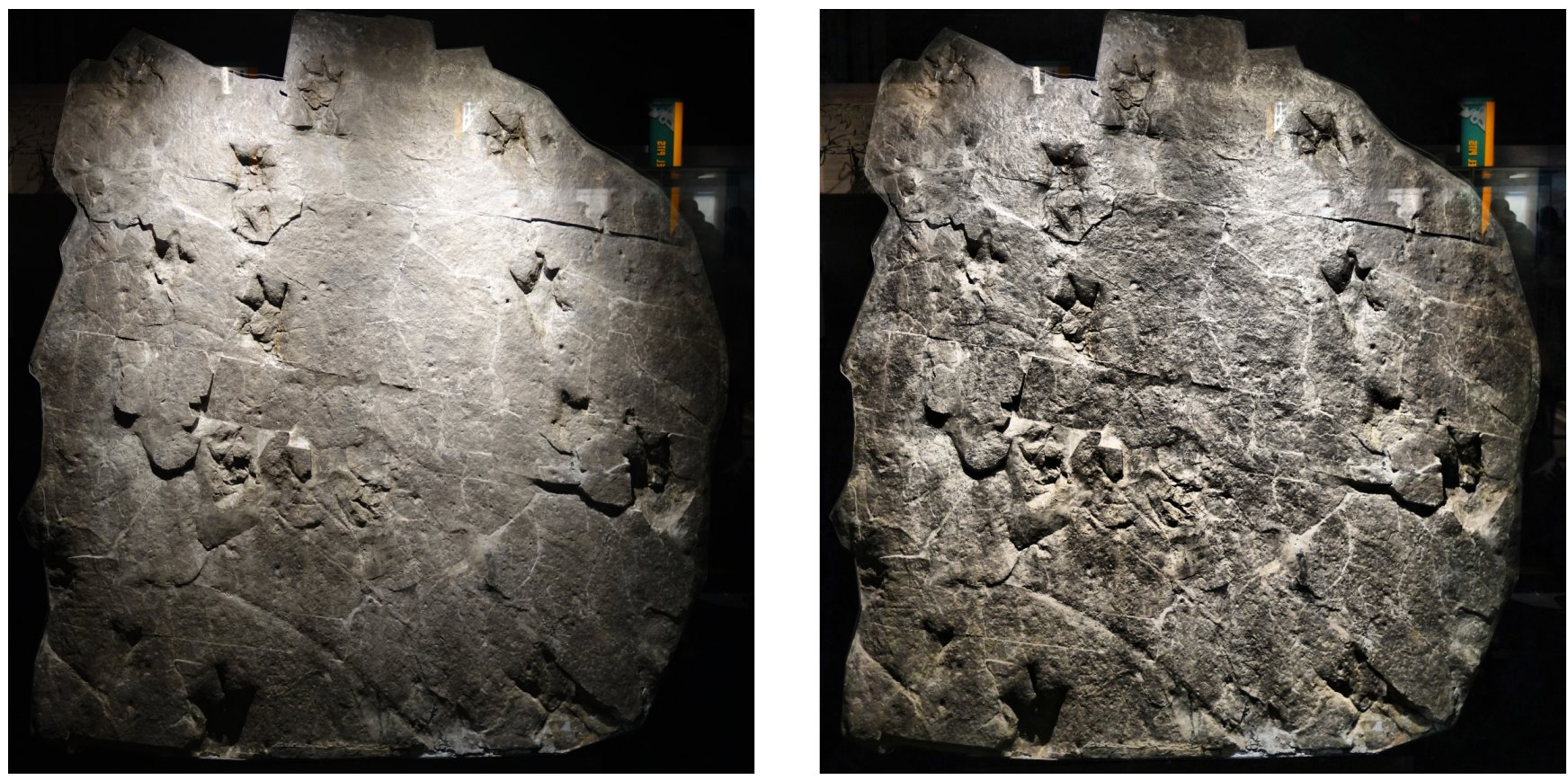

Fig. 2: Contrast limited adaptive histogram equalisation (CLAHE); left: original photograph of theropod tracks on display at the Royal Tyrrell Museum of Palaeontology, Drumheller, Alberta; right: same photograph processed with CLAHE. Note that bright and dark regions were modulated.

\section{Orthophotos}

Orthophotos are geometrically corrected (orthorectified) photographs of an area in top view (Fig. 3A, 4A). They can be extracted from 3D models using MeshLab or ParaView, provided that texture files were included with the model, or calculated directly from the aligned images using Metashape. In MeshLab and ParaView, the default textured model view will also include shading according to a virtual light source and, if present, vertex colours. To generate a pure orthophoto without additional oblique light shading, the virtual light source (ParaView) or vertex colours and model shading (MeshLab) have to be disabled. 

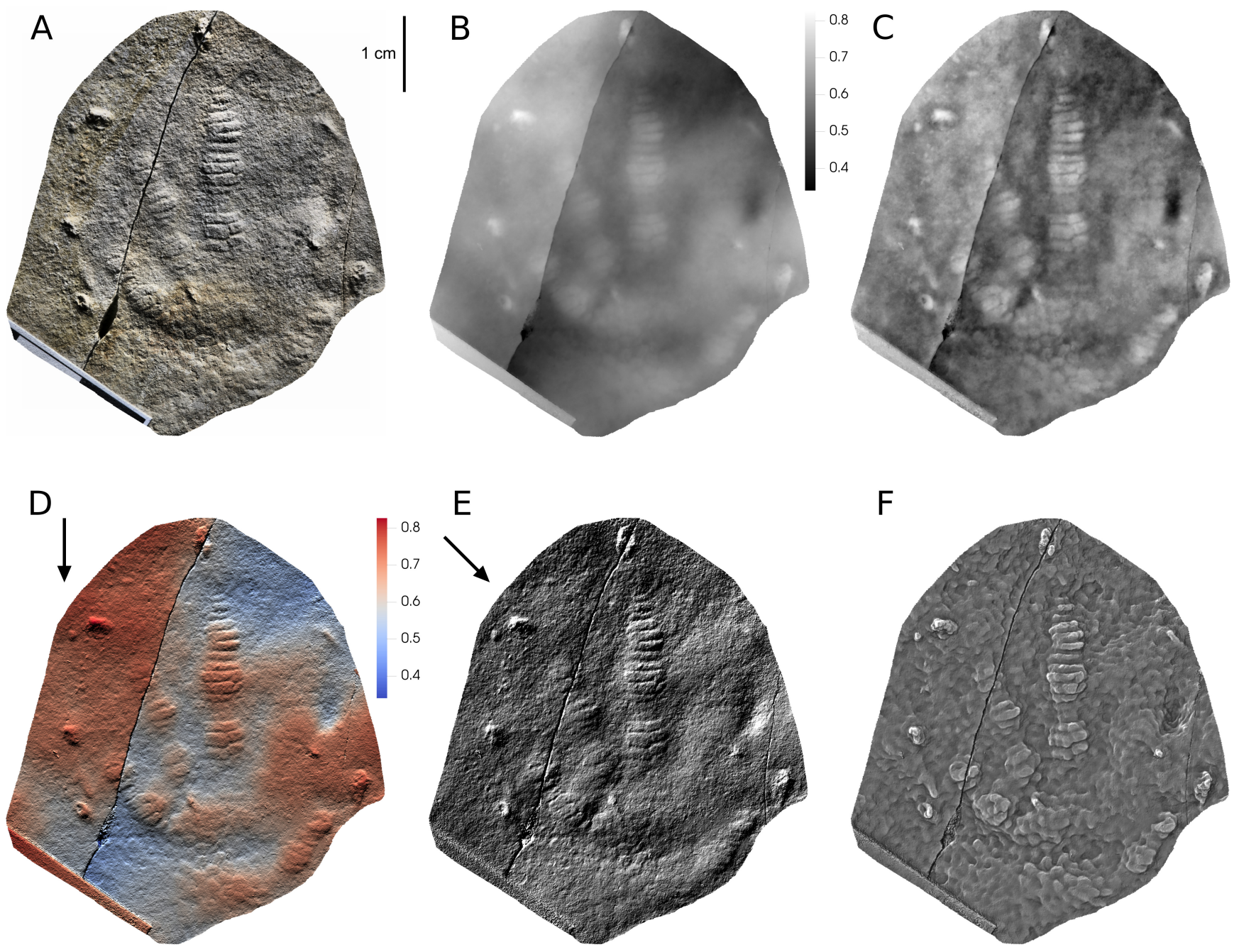

Fig 3: Different graphical representations of Rhynchosauroides footprint 6067a from Bernburg, Germany (collection Frank Trostheide), with very low relief but well-preserved skin impressions. All plots except for $(F)$ generated with ParaView. A: Orthophoto, enhanced with CLAHE, without oblique light shading. B: Greyscale heightmap, without oblique light shading. C: The plot of B processed with CLAHE. Note that individual scales are better visible, but that shades of grey do not correspond to specific heights anymore. D: Heightmap blue-to-red with virtual low-angled light shading. E: Shaded relief, virtual light source from the top left. $F$ : Radiance scaling plot, enhancing the appearance of scale impressions. To scale. Colour legends in $\mathrm{cm}$. Arrows indicate direction of low-angled light. 


\section{Height maps}

Height maps, also referred to as "depth-colour maps", are colour-coded elevation plots that allow for the effective illustration of the depth profile of a surface model. In many cases, height maps convey the 3D morphology of tracks more clearly and detailed than simple photographs, and may even highlight features that are difficult to see in the field. Height maps can be generated using ParaView, CloudCompare, or Metashape Professional.

ParaView and CloudCompare provide a selection of colour scales (i.e., the selection and number of separate colours to be used), and furthermore allow to import custom-made ones. As cool colours are generally interpreted as depths and warm colours as heights, the colour scale of choice should reflect this rule. The optimal colour scale to use depends on the respective model. Simple colour scales with few colours, such as the default colour scale of ParaView ("Cool to Warm"; blue-white-red), work well with most models and are easy to interpret, although the low amount of colours may not sufficiently differentiate between subtle changes in relief, so that details may get lost or are difficult to see from the height map. On the other hand, colour scales with more colours will show more detail, but are often more difficult to interpret especially when the relief of the tracks is smaller than the relief of the surrounding surface. In the latter case, separate footprints and regions of the surface may appear in different colours, making visual comparisons of these footprints and areas difficult. An example of a colour scale with many colours that has been tested in previous studies is the "Rainbow Desaturated" preset of ParaView, which includes eight distinct colours (Fig. 4B). As an alternative, the greyscale preset running from white to black (or vice versa) can be used (Fig. 3B). This preset is especially advantageous in cases where the resulting figure cannot be printed in colour. As is the case with colour scales that include few distinct colours, however, details of the relief may get lost.

Per default, the colour scale will include the entire depth of the model. It may, therefore, be necessary to tight-crop the model to remove surroundings such as slab borders to decrease the total relief, so that the objects of interest (i.e., the trace fossils) are better differentiated (see above for cropping methods). Alternatively or additionally, the highest and/or lowest portions of the model may be filled with uniform colour. The colour distribution can also be 
adjusted manually to concentrate more colours within the height interval of interest, possibly improving the visibility of the details of interest.

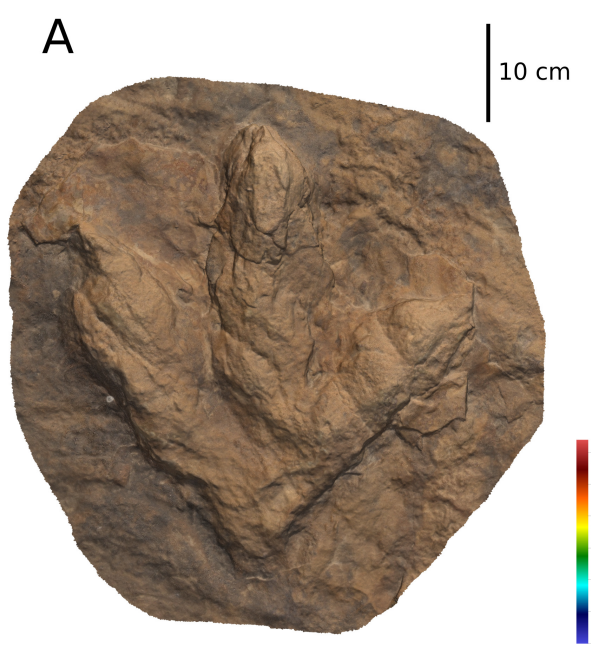

B
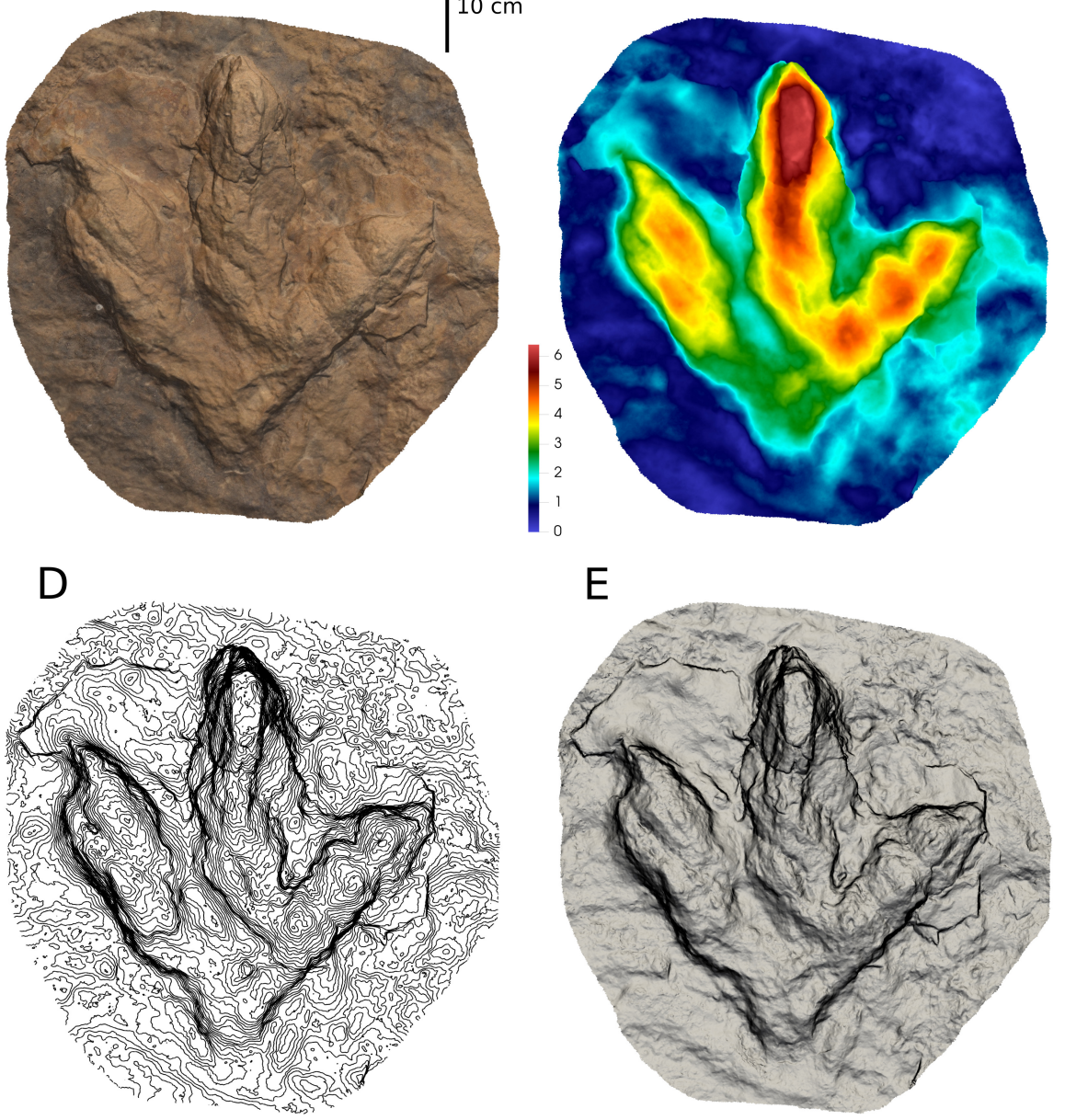

$\mathrm{E}$

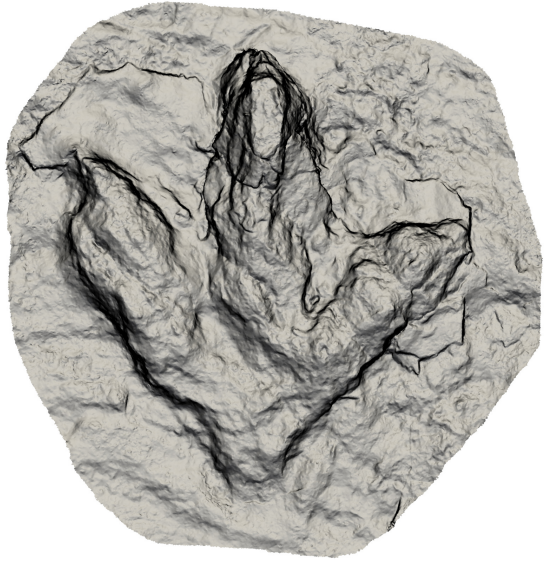

C

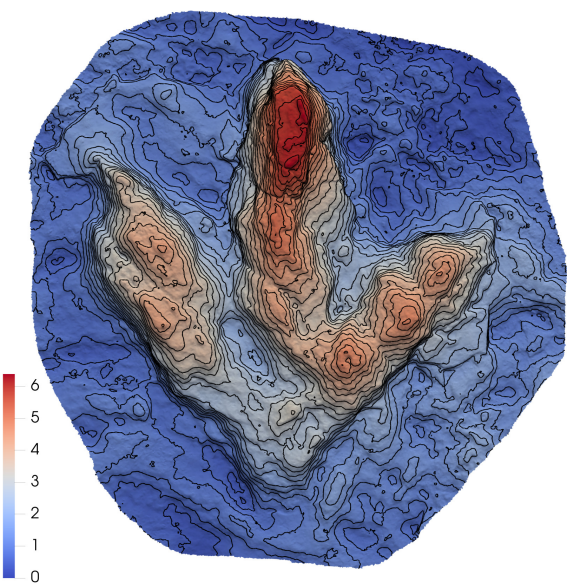

$\mathrm{F}$

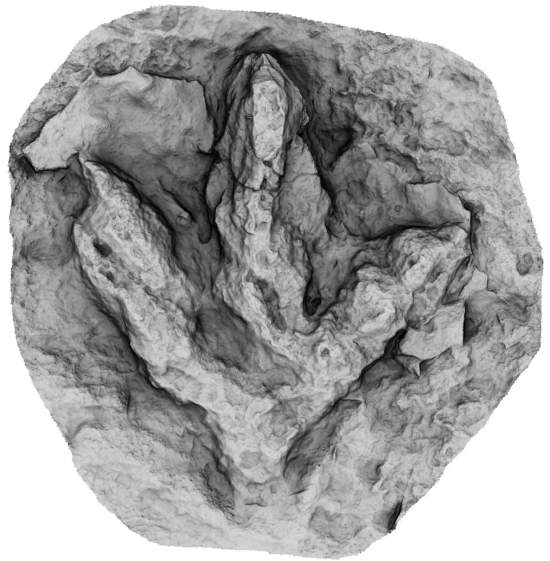

Fig. 4: Natural cast of T3/18, footprint of a large theropod trackway from Münchehagen, Germany (Lallensack et al., 2016). A: Orthophoto combined with oblique light shading. B: Heightmap of multiple colours (rainbow) without oblique light shading. C: Height map (blue-tored) combined with contour lines $(n=30)$ and oblique light shading. D: High number of contours $(n=50)$ to highlight surface steepness. E: Inclination plot highlighting steepness combined with oblique light shading. F: Ambient occlusion plot enhancing surface detail. To scale. Colour legends in $\mathrm{cm}$. 
Both ParaView and CloudCompare combine the height map with shading according to a virtual light source per default. While both software solutions allow for adjusting the position of this light source, ParaView allows for adjusting the intensity and warmth. Adjusting these values can improve the visibility of subtle surface features; especially the option to lower the light source to produce low-angled light can be advantageous (Fig. 3D; see section "shaded reliefs" below). When fine surface detail is not of interest, the oblique light shading can be disabled, resulting in a clearer visualisation of larger relief features (Fig. 3B, 4B). Disabling oblique light shading to achieve consistent illumination of edges is especially important when the margins of shape features (i.e., their dimensions and shape) need to be visualised accurately.

A height map requires both a regular scale and a colour legend that indicates the heights associated with the colours. Colour legends can be displayed by both ParaView and CloudCompare.

\section{Contour plots}

Contour plots, consisting of lines of equal elevation, are a common way of displaying threedimensional topography in two dimensions, especially single footprints. The contour interval can either be relative to the total height of the model (using a fixed number of contours) or absolute (e.g., fixed intervals of $0.5 \mathrm{~cm}$ ). Relative contour intervals come with the advantage that the same number of contours can be used for footprints of different sizes. This allows for comparing and measuring specific contours between specimens. Contour plots can be generated with ParaView using the "Contour" function and with CloudCompare using the "Rasterize" tool (see Appendix for instructions). Contour plots can also be created with Metashape Professional.

Attributes that frequently need to be adjusted are the colour and line thickness. With both ParaView and CloudCompare, it is possible to colour the individual contours by height using the selected colour scale. A contour plot may be combined with any of the other graphical representations that may be created with ParaView or CloudCompare. A common approach is 
to combine contour plots with height maps. Combining separate contour plots allows for different formats (e.g., to highlight each fifth contour using a different colour and line width).
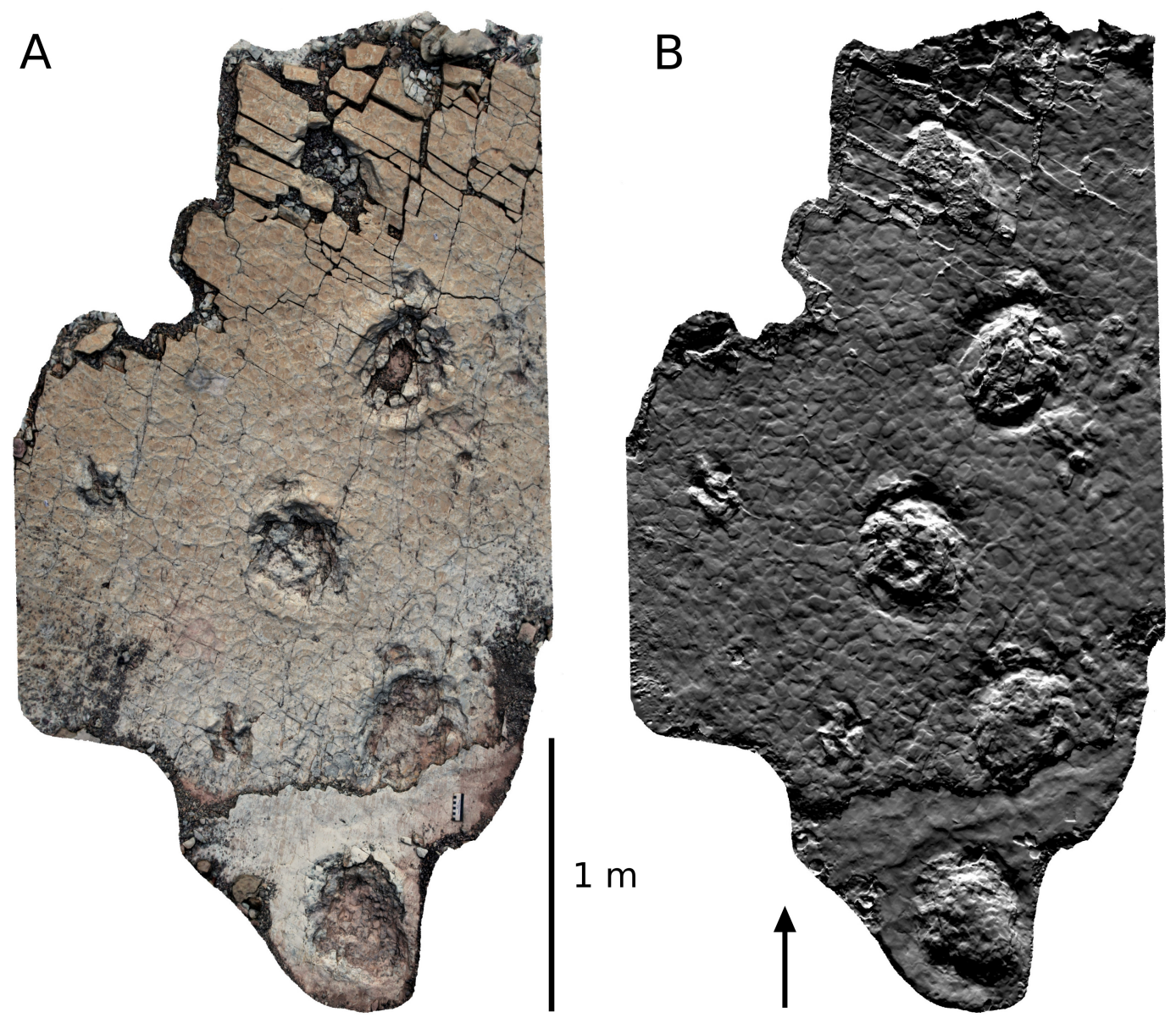

Fig. 5: Sauropod trackway from the Late Triassic of Greenland (Lallensack et al., 2017). A: Orthophoto. B: Shaded relief, with the virtual light set from below to highlight pedal claw impressions; note that the footprints may be perceived as convex hyporeliefs due to the position of the light source (light direction indicated by arrow).

\section{Shaded reliefs}

Shaded reliefs make use of an virtual light source positioned at an oblique angle to the model surface to emphasize the steepness of the topography. Such shading is often used in 
combination with other forms of visualisation, such as height maps (Fig. 3D; 4C) and orthophotos (Fig. 4A). However, when the relief of the model is low, shaded reliefs are most effective in enhancing subtle surface detail when used with any colour information disabled and with the virtual light at a very low angle (Fig. 3E, 5B). As the digital counterpart to the respective method in real-world photography, shaded reliefs are intuitive to use, and suitable for greyscale figures.

The optimal position of the light source depends on the respective surface features that shall be enhanced. Low-relief surfaces generally work best with a very low position of the light source, while higher reliefs require higher light source positions. Lighting from the upper left is the standard used for scientific illustrations, and therefore most easy to interpret by the human eye. Other light source positions may be required to highlight surface details of interest (e.g., claw impressions), depending on the orientation of these structures (Fig. 5B). Light from the bottom, however, may cause concavities to appear as convexities and vice versa (Fig. 5B). MeshLab, ParaView, and CloudCompare allow for moving the light source interactively.

\section{Surface inclination plots}

It is possible to shade the mesh surface according to its inclination relative to the horizontal plane (i.e., the orientation of the vertex normals relative to the z-axis). In such plots, white indicates a more horizontal, and black a more vertical surface. With this technique, the slopes of the surface can be effectively enhanced (Fig. 4E). This may also help with detecting and tracing the steepest slopes along the footprint margin for measurements and interpretive outlines; as the slopes can be displayed with high contrast, such plots may furthermore serve as objective replacements for interpretive outline drawings. Inclination plots can be easily created with ParaView by setting the mesh colouring to "Normals", restricting the range of the colour scale to increase contrast, and by choosing a black-to-white scale. The use of colour scales is possible but only greyscales will provide the illusion of virtual lighting and are easy to interpret by the human eye. Similar plots may also be achieved by using a very high number of contours lines. 
Inclination plots appear similar to a shaded relief, but without depending on the lighting. This is an advantage when the relief of the surface is more pronounced, as it is often the case with a single footprint. With low-relief surfaces, in contrast, enhancement of surface noise may obscure the features of interest, and directed light (shaded reliefs) is the more appropriate method to use.

It is possible to choose the $x$ or $y$-axis instead of the z-axis. In this case, the resulting plot can be largely equivalent to a shaded relief, with the perceived lighting being parallel to the selected axis, and the perceived angle of lighting determined by the narrowness of the colour scale. Such inclination plots can, therefore, be a quick way to produce shaded reliefs, enhancing detail on low-relief surfaces.

\section{Ambient occlusion}

In ambient occlusion (Zhukov et al., 1998), each point of the surface is shaded according to its exposure to ambient lighting, i.e. the portion of "sky" visible from the respective point. More exposed parts of the surface (e.g., at the top of a projection) will appear brighter, while more occluded parts (e.g., adjacent to a projection) will appear darker. Ambient occlusion is used to effectively enhance surface detail, and improve the perception of 3D morphologies (Fig. 4F). For surfaces with higher relief, surface detail may appear clearer than with any of the other methods presented herein. The method is, however, not suitable for low-relief models. It has been, to our knowledge, first applied to ichnofossils by Ahn et al. (2010). Ambient occlusion plots can be generated with MeshLab, CloudCompare, and Metashape Professional.

\section{Radiance scaling}

The visualisation of 3D models is an active field of research, and additional, more elaborated methods that enhance the appearance of surface features selectively based on size and morphology have been published. Amongst these promising techniques is radiance scaling 
(Vergne et al., 2010), which uses the intensity of reflected light to improve the distinction between convex and concave surface features of a specified size. This method may be used to detect and characterise shallow impressions, such as very faint footprints or skin impressions, and may provide detail not readily visible otherwise (Fig. 3F). Radiance scaling has been implemented in MeshLab, but this implementation does not allow for setting the scale of the features to be detected and is therefore of limited use for visualising ichnofossils. Full functionality is provided by the free research software Gratin (Vergne and Barla, 2015), for which a ready-made pipeline is provided in the supplementary information of this paper, with detailed instructions given in the appendix.

\section{Cross sections}

Cross sections, i.e. height differences along a pre-defined line on the model surface, may be helpful to study relief structures such as skin impressions. By simplifying the threedimensional structure to a two-dimensional line, cross sections can illustrate the precise shape of structures (Fig. 6); their xy-coordinates can also be exported to quantify and analyse these shapes. Cross sections can be produced with the "Extract Sections / Unfold" tool of CloudCompare, as well as with Metashape Professional. 


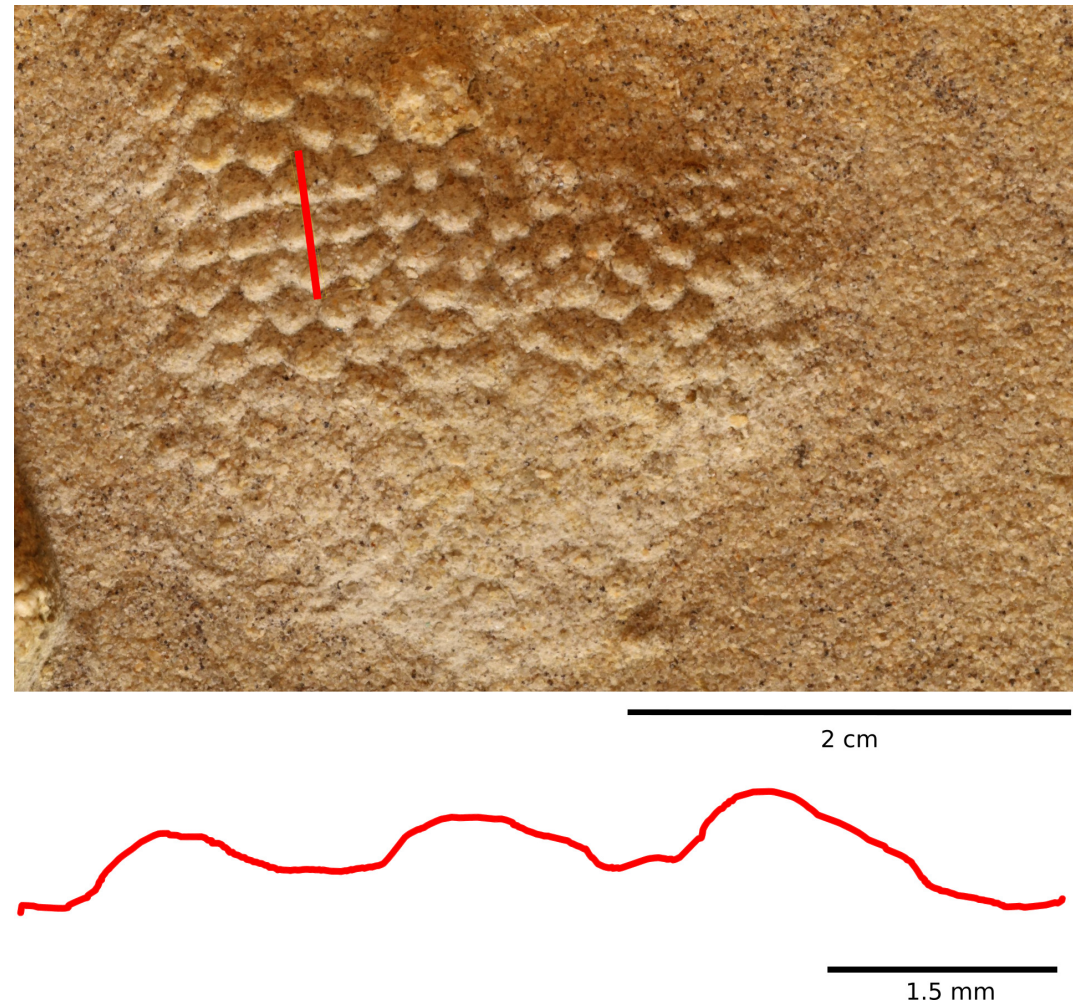

Fig. 6: Obtaining cross profiles. Top: Orthophoto of a 3D model of a Synaptichnium track from Berndorf, Germany (SKF UMBD 138, Collection H. Klein, Saurierwelt Paläontologisches Museum, Neumarkt, Germany; see Klein and Lucas, 2017) showing skin impressions, with the red line indicating the extracted profile. Bottom: Elevation profile of the three subsequent tubercle impressions.

\section{DATA EXTRACTION}

\section{Measurements and line drawings}

Provided that the model is scaled (see "Scaling" above), it can be used for detailed measurements. The discussed software all provide tools that allow for measuring in 3D directly on the model, and are useful for any measurements that are not necessarily parallel to the horizontal plane of the model. In the majority of cases, however, measurements need to 
be taken parallel to the horizontal plane, and accidental deviations of the measured line from the horizontal plane may result in significant error. These deviations are difficult to avoid as the starting and end points of the measured line have to be placed on the three-dimensional mesh surface, making it necessary to set the $z$ value to equal values after defining the line.

We here recommend taking measurements from exported 2D-visualisations of the model in an external software such as Inkscape. This effectively prohibits measurements in a plane other than the horizontal plane, and offers more flexibility and additional functions such as angular measurements and helper lines. In Inkscape, measurements can be done using the "Measurement tool" (see detailed instructions in the appendix).

We recommend the use of vector graphic editors such as Inkscape for producing interpretive tracings of footprints, trackways, and whole tracksites. It is possible to consider multiple 2D visualisations together by placing each visualisation on a separate layer in the vector editor. The different visualisations can then be aligned on top of each other into an image stack. The tracing itself is done on an additional transparent top layer, and the visualisations of the lower layers can be easily switched on and off for reference while drawing.

\section{Automated calculation of trackway parameters based on xy-coordinates}

If a large number of measurements is needed, especially multiple parameters of trackways, it can be efficient to collect xy-coordinates from which linear and angular measurements can be automatically calculated afterwards (Lallensack et al., 2019). Our aim is to make these methods as accessible as possible by providing a detailed procedure in the appendix, and the spreadsheet "trackway calculator" for the automated calculation of a broad array of parameters of quadrupedal trackways in the supplementary material.

2D coordinates are here collected using Inkscape, although different software solutions (such as Image J) may be used alternatively. Coordinate export in Inkscape requires the installation of the plugin "ExportXY" that is also provided in the supplementary information of this paper. 2D coordinates may be collected from interpretive outline drawings or 2D-visualisations of the 3D model. The "trackway calculator" sheet expects five coordinates per footprint (Fig. 7), 
A

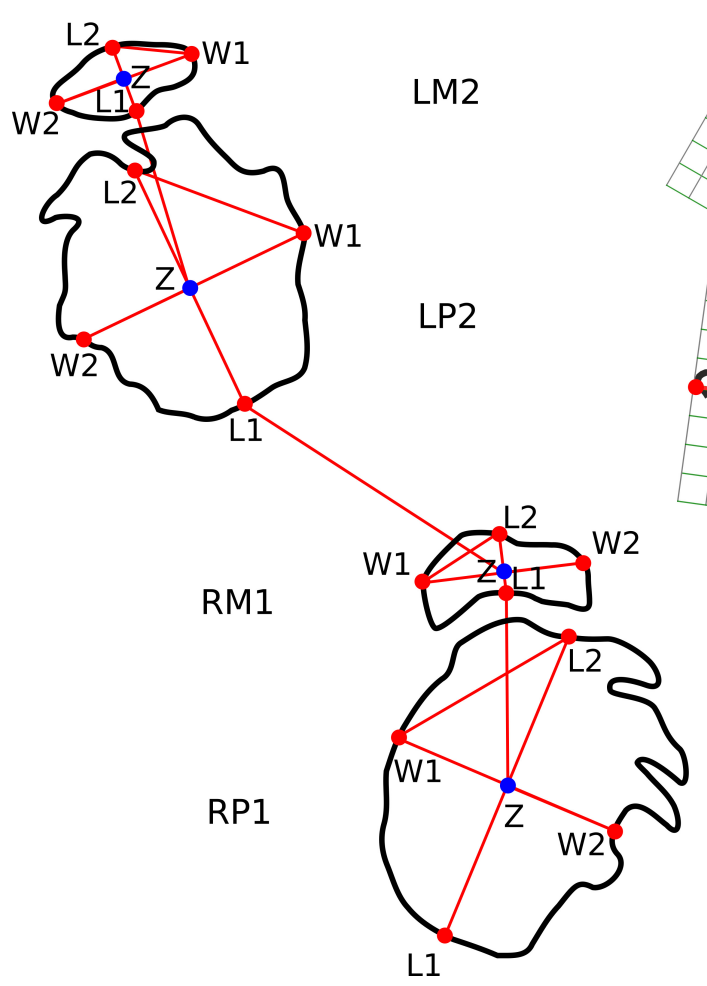

B

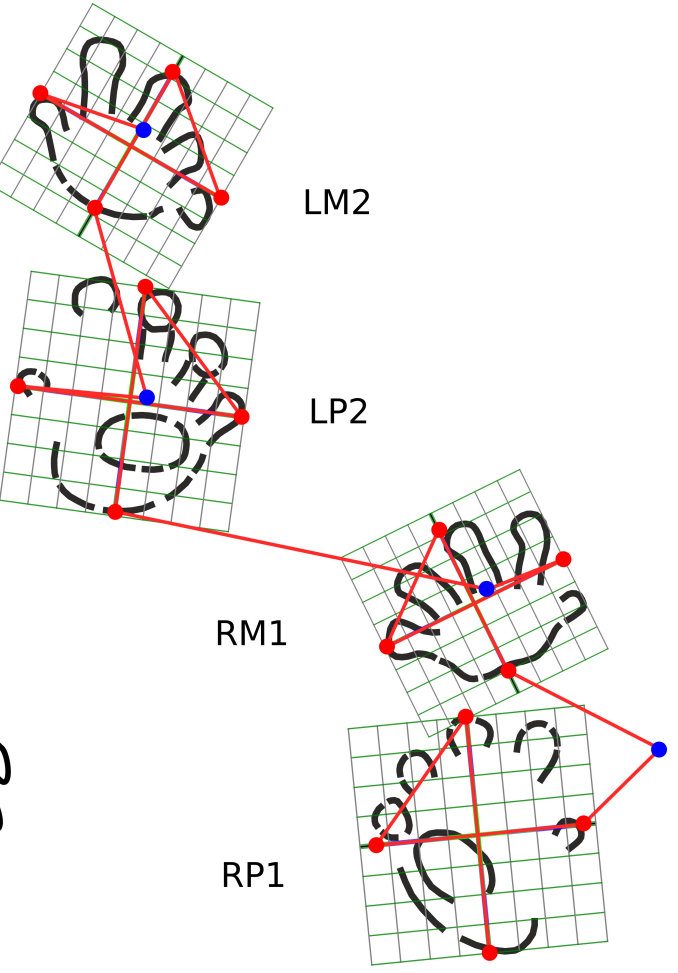

Fig. 7: Sequential 2D-coordinates (five per footprint) extracted using Inkscape for the automatic calculation of trackway parameters with the "Trackway Calculator" spreadsheet. The trackway reference points ("Z") are shown in blue. A: Sauropod trackway S1 from the Late Triassic of Greenland (Lallensack et al., 2017), with individual coordinate points labeled, and trackway reference points coinciding with the intersection of the lines defining the footprint length and width. B: Diadectomorph trackway (Ichniotherium cottae) from Birkheide Germany (Buchwitz and Voigt, 2018), showing the use of fitted templates for accurate coordinate extraction according to the footprint rotation. Trackway reference points are defined as the basis of digit impression III. Note that the reference coordinate cannot be placed for footprint RP1 given the incomplete digit impression III, and is moved to the right to be automatically excluded by the calculation spreadsheet. See text for details. 
which have to be placed in the following order: L1 and L2 mark the posterior and anterior end of the trackway midline, respectively, and capture footprint length and rotation. W1 and W2 are the medial and lateral points of a line perpendicular to the trackway midline, and capture footprint width. The last coordinate, $Z$, is the reference point used for the calculation of distances between footprints (such as pace and stride length). $Z$ can simply be the intersection between the L1-L2 and W1-W2 lines, but other definitions (e.g., the tip of one of the digit impressions) may be chosen.

Besides the coordinates, the "trackway calculator" sheet accepts additional data: The ID is a unique identification name or number of the trackway. The scale can be easily added by extracting the coordinates of the beginning and end of the scale bar in Inkscape. The "Hipheight - Pes length ratio" is required for calculations of absolute locomotion speed; e.g., a value of " 4 " is a commonly assumed (Alexander, 1976). The optional "Cardinal direction" field accepts coordinates of a line capturing the south-to-north direction.

The output for each individual footprint can be found when scrolling to the right in the first sheet. A summary is presented in the second sheet and includes the average, median, standard variation, coefficient of variation, minimum, and maximum of different parameters; the mean configuration and the standardised mean configuration; and statistical tests for selected pairs of measurements (t-tests for the equality of means and f-tests for the equality of variances). Parameters per step cycle can be found on the third sheet. Trackway parameters are calculated using both standard trigonometric functions and vectors. Footprint rotation angles are calculated based on the preceding, following, and opposite stride for maximum accuracy.

\section{D data extraction for analysis in $R$}

Methods for data extraction described above are limited to interpretive tracings, which are partly subjective (Falkingham, 2016), or single measurements, which may not capture the complete shape. Contour lines provide a natural basis for more comprehensive shape analyses of footprints. ParaView allows for exporting point coordinates of contour plots 
("Filters" > "Integrate Variables"); these coordinates are however difficult to use as they are neither ordered nor sorted by contour. Alternatively, 3D models may be directly imported into the free statistical computing environment $R$ (R Core Team, 2019) using the vcglmport function of the Rvcg package (Schlager, 2017), which supports a number of common formats including PLY. Contour lines may be extracted from the imported model using the "getContourLines" function of the contoureR R package (Hamilton, 2015). Methods for processing these contours for ichnological studies were presented by Lallensack (2019), including their sorting, resampling, filtering, and homologisation as well as the automatic derivation of outlines, landmarks, and measurements.

\section{CONCLUSIONS}

A comprehensive methodology is presented to produce, process, and analyse threedimensional surface models for ichnological research using free and open-source software. We note that the production of quality photogrammetric models is not trivial, and review the most common mistakes in obtaining pictures. The presented methods for data extraction require accurate model scaling, automatic fitting to the horizontal plane, and orthographic projection. 3D models allow for 2D visualisations such as orthophotos, height maps, shaded reliefs, contour plots, inclination plots, ambient occlusion, and radiance scaling, which can effectively enhance different aspects of the 3D morphology and provide a broader scope than traditional visualisation techniques such as photographs. The different visualisation techniques are compared, and recommendations on method choice for different purposes and types of data given. We provide a method for the automatic calculation of trackway parameters based on xy-coordinates collected from 2D visualisations of the models. It is argued that, in many cases, the discussed approaches have the potential to replace respective traditional approaches that previously had to be carried out in the field. 


\section{SUPPLEMENTARY MATERIAL}

The trackway calculator spreadsheet, the ExportXY-plugin for Inkscape, and example files are available at:

https://doi.org/10.6084/m9.figshare.11739897.v1

A Gratin pipeline for radiance scaling by Romain Vergne is available at:

https://doi.org/10.6084/m9.figshare.12251966.v2

\section{ACKNOWLEDGEMENTS}

We thank Peter Falkingham for comments on an early version of the manuscript, and Brent Breithaupt and an anonymous reviewer for their helpful comments during peer review. Ikuko Tanaka is thanked for testing the presented software instructions. We thank Romain Vergne for help with applying radiance scaling and providing the software pipeline available in the supplementary information, Heinrich Mallison for advice regarding photogrammetry of small objects, Hendrik Klein for access to specimens, and John Cliff for his efforts to help publishing the export.xy plugin for Inkscape under a free license.

\section{REFERENCES CITED}

Ahn, J., K.-Y. Wohn, and D.-Y. Kong. 2010. 3D digital documentation of dinosaur footprints. 2010 16th International Conference on Virtual Systems and Multimedia, p. 340-345.

Agisoft LLC. 2020. Agisoft Metashape User Manual: Professional Edition, Version 1.6. https://www.agisoft.com/downloads/user-manuals/

Alexander, R. McN. 1976. Estimates of speeds of dinosaurs. Nature 261:129-130. 
AliceVision. 2018. Meshroom: A 3D Reconstruction Software.

https://github.com/alicevision/meshroom

Ayachit, U. 2019. The ParaView Guide. https://www.paraview.org/paraview-guide/

Bates, K. T., F. Rarity, P. L. Manning, D. Hodgetts, B. Vila, O. Oms, À. Galobart, and R. L. Gawthorpe. 2008. High-resolution LiDAR and photogrammetric survey of the Fumanya dinosaur tracksites (Catalonia): implications for the conservation and interpretation of geological heritage sites. Journal of the Geological Society 165:115-127.

Belvedere, M., M. R. Bennett, D. Marty, M. Budka, S. C. Reynolds, and R. Bakirov. 2018. Stattracks and mediotypes: powerful tools for modern ichnology based on 3D models. PeerJ 6:e4247.

Breithaupt, B. H., N. A. Matthews, and T. A. Noble. 2004. An integrated approach to threedimensional data collection at dinosaur tracksites in the Rocky Mountain West. Ichnos 11:1126.

Buchwitz, M., and S. Voigt. 2018. On the morphological variability of Ichniotherium tracks and evolution of locomotion in the sistergroup of amniotes. PeerJ 6:e4346.

Carbonneau, P. E., S. N. Lane, and N. E. Bergeron. 2003. Cost-effective non-metric close-range digital photogrammetry and its application to a study of coarse gravel river beds. International Journal of Remote Sensing 24:2837-2854.

Cignoni, P., M. Callieri, M. Corsini, M. Dellepiane, F. Ganovelli, and G. Ranzuglia. 2008. Meshlab: an open-source mesh processing tool. Eurographics Italian Chapter Conference 2008:129-136.

CloudCompare. 2019. GPL software. https://cloudcompare.org/

Falkingham, P. L. 2012. Acquisition of high resolution three-dimensional models using free, opensource, photogrammetric software. Palaeontologia Electronica 15:1-15.

Falkingham, P. L. 2016. Applying Objective Methods to Subjective Track Outlines; pp. $72-81$ in P. L. Falkingham, D. Marty, and A. Richter (eds.), Dinosaur Tracks: The Next Steps. Indiana University Press, Bloomington.

Falkingham, P. L., K. T. Bates, and J. O. Farlow. 2014. Historical photogrammetry: Bird's Paluxy River dinosaur chase sequence digitally reconstructed as it was prior to excavation 70 years ago. PLoS ONE 9:e93247. 
Falkingham, P. L., K. T. Bates, M. Avanzini, M. Bennett, E. M. Bordy, B. H. Breithaupt, D. Castanera, P. Citton, I. Díaz-Martínez, J. O. Farlow, A. R. Fiorillo, S. M. Gatesy, P. Getty, K. G. Hatala, J. J. Hornung, J. A. Hyatt, H. Klein, J. N. Lallensack, A. J. Martin, D. Marty, N. A. Matthews, C. A. Meyer, J. Milàn, N. J. Minter, N. L. Razzolini, A. Romilio, S. W. Salisbury, L. Sciscio, I. Tanaka, A. L. A. Wiseman, L. D. Xing, and M. Belvedere. 2018. A standard protocol for documenting modern and fossil ichnological data. Palaeontology 61:469-480.

Hamilton, N. 2015. contoureR: Contouring of Non-Regular Three-Dimensional Data. https:/CRAN.Rproject.org/package $=$ contoureR

Inkscape. 2020. Inkscape: Draw Freely. Version 1.0. https://inkscape.org/

Klein, H., and S. G. Lucas. 2018. Diverse Middle Triassic tetrapod footprint assemblage from the Muschelkalk of Germany. Ichnos 25:162-176.

Lallensack, J. N. 2019. Automatic generation of objective footprint outlines. PeerJ 7:e7203.

Lallensack, J. N., A. H. van Heteren, and O. Wings. 2016. Geometric morphometric analysis of intratrackway variability: a case study on theropod and ornithopod dinosaur trackways from Münchehagen (Lower Cretaceous, Germany). PeerJ 4:e2059.

Lallensack, J. N., P. M. Sander, N. Knötschke, and O. Wings. 2015. Dinosaur tracks from the Langenberg Quarry (Late Jurassic, Germany) reconstructed with historical photogrammetry: Evidence for large theropods soon after insular dwarfism. Palaeontologia Electronica 18.2.24A:1-34.

Lallensack, J. N., S. Ishigaki, A. Lagnaoui, M. Buchwitz, and O. Wings. 2019. Forelimb orientation and locomotion of sauropod dinosaurs: insights from the Middle Jurassic Tafaytour Tracksite (Argana Basin, Morocco). Journal of Vertebrate Paleontology 5:1-18.

Lallensack, J. N., H. Klein, J. Milàn, O. Wings, O. Mateus, and L. B. Clemmensen. 2017. Sauropodomorph dinosaur trackways from the Fleming Fjord Formation of East Greenland: evidence for Late Triassic sauropods. Acta Palaeontologica Polonica 62:833-843.

Mallison, H., and O. Wings. 2014. Photogrammetry in paleontology - A practical guide. Journal of Paleontological Techniques 12:1-31.

Matthews, N. A. 2008. Aerial and Close-Range Photogrammetric Technology: Providing Resource Documentation, Interpretation, and Preservation. Technical Note 428. U.S. Department of the Interior, Bureau of Land Management, National Operations Center, Denver, Colorado, pp. 42. 
Matthews, N. A., and B. H. Breithaupt. 2001. Close-range Photogrammetric Experiments at Dinosaur Ridge. The Mountain Geologist 38:147-153.

Matthews, N. A., T. Noble, and B. H. Breithaupt. 2006. The application of photogrammetry, remote sensing and Geographic Information Systems (GIS) to fossil resource management. Americas Antiquities: 100 Years of Managing Fossils on Federal Lands: Bulletin 34:119-131.

Matthews, N. A., T. Noble, and B. H. Breithaupt. 2016. Close-Range Photogrammetry for 3-D Ichnology: The Basics of Photogrammetric Ichnology; pp. 28-55 in P. L. Falkingham, D. Marty, and A. Richter (eds.), Dinosaur Tracks: The Next Steps. Indiana University Press.

R Core Team. 2019. R: A Language and Environment for Statistical Computing. R Foundation for Statistical Computing, Vienna, Austria. http://www.r-project.org/

Santella, M., and A. R. Milner. 2017. Coupling focus stacking with photogrammetry to illustrate small fossil teeth. Journal of Paleontological Techniques, 18:1-17.

Schlager, S. 2017. Morpho and Rvcg - Shape Analysis in R; pp. 217-256 in G. Zheng, S. Li, and G. Szekely (eds.), Statistical Shape and Deformation Analysis. Academic Press, Cambridge, Massachusetts. 508 pp.

Thulborn, R. A. 1990. Dinosaur Tracks. Chapman and Hall, London, New York, 410 pp.

Vergne, R., and P. Barla. 2015. Designing gratin, a gpu-tailored node-based system. Journal of Computer Graphics Techniques 4:54-71.

Vergne, R., R. Pacanowski, P. Barla, X. Granier, and C. Schlick. 2010. Radiance scaling for versatile surface enhancement. Proceedings of the 2010 ACM SIGGRAPH Symposium on Interactive 3D Graphics and Games, p. 143-150.

Wings, O., J. N. Lallensack, and H. Mallison. 2016. The Early Cretaceous Dinosaur Trackways in Münchehagen (Lower Saxony, Germany) - 3D photogrammetry as basis for geometric morphometric analysis of shape variation and evaluation of material loss during excavation; pp. 56-71 in P. L. Falkingham, D. Marty, and A. Richter (eds.), Dinosaur Tracks: The Next Steps. Indiana University Press, Bloomington.

Zhukov, S., A. Iones, and G. Kronin. 1998. An ambient light illumination model. Rendering Techniques '98, p. 45-55.

Zuiderveld, K. 1994. Contrast limited adaptive histogram equalization. Graphics Gems IV, p. 474485. 


\section{APPENDIX: SOFTWARE INSTRUCTIONS}

\section{Online tutorials}

ParaView tutorial videos illustrating methods discussed in this paper by Jens N. Lallensack: https://www.youtube.com/playlist?list=PLj3Ekg0ZUJ-JCSvE4W53lhe282N9vUOfZ

Collection of photogrammetry tutorials by Heinrich Mallison: Dinosaurpalaeo https://dinosaurpalaeo.wordpress.com/

Collection of photogrammetry tutorials by Peter Falkingham: https://peterfalkingham.com/blog/

Collection of MeshLab introductory videos: Mister P. MeshLab Tutorials https://www.youtube.com/playlist?list=PL8B1E816EAE236B4D

\section{Model generation}

\section{Meshroom}

In Meshroom, images first need to be added to the "Images" pane (using drag\&drop), after which the different steps (displayed as "nodes" in the "Graph Editor" pane) can be run automatically with default values by pressing the "Start" button. Results can be viewed in the "3D Viewer" pane by double-clicking on the respective item in the "Graph Editor" (e.g., "MeshFiltering" for the basic mesh). Results, including .obj files, from all nodes will be automatically exported to the folder where the project was saved. While the default options lead to decent results in many cases, processing parameters may be changed using the "Graph Editor". Model processing may fail if minimum requirements of the default settings on hardware resources and image quality and quantity are not met. 


\section{Metashape}

In Metashape, the model is run by adding photos using the "add photos" icon of the "Workspace" pane, and calling the four principal steps in succession from the "Workflow" menu. As in Meshroom, the alignment accuracy may be set to "high" or even "highest" for best quality in the "Align Photos" step. In case the alignment is inaccurate, it can help to disable the "generic preselection" option and/or to drastically increase the "tie points" and "key points" in the "Align Photos..." dialog.

To reduce noise in the final model, it is advisable to remove less accurate points from the sparse cloud before continuing with dense model generation. This can be done using the "gradual selection" function available from the "Model" menu, where the "reconstruction uncertainty" filter, used with a threshold of "10", is especially effective. The points selected by the filter should then be deleted, and the alignment optimised by calling "Tools" > "Optimize cameras", checking "Adaptive camera model fitting". We furthermore recommend to set the "depth filtering" option of the "Build dense cloud..." dialog to "aggressive". This may result in the loss out very small-scale differences in relief, which, however, in the case of ichnological fossils are much more likely to represent noise than actual features.

\section{Model post-processing}

The three post-processing steps (cropping, scaling, and orienting) can be done in MeshLab. Note that in MeshLab the model must be exported to save the changes ("File" > "Export Mesh"). To allow for generating surface inclination plots in ParaView (see below), the "Normal" option needs to be activated in the "Choose Saving Options" dialogue that will appear when attempting to save a model in MeshLab.

\section{Cropping}

- "Select Faces in a rectangular region" tool, available from the toolbar: Selections can be inverted by pressing the "i" key, and deleted using the "Delete the current set of selected faces and all the vertices surrounded by that faces" key, which is the 
rightmost button of the tool bar. Simply hitting the "del" or "delete" key will only remove the faces but not the vertex points.

- "Select Faces/Vertices inside polyline area" tool. As above; it has to be ascertained that "Faces Selection" (not "Vertices Selection") is active (see instructions in the red window that will appear in the upper left of the screen after calling the tool).

- "Remove isolated pieces (wrt Diameter)" filter, available in "Filters" > "Cleaning and repairing": A value of, e.g., "50" in the "perc on" field in the filter dialog removes all disconnected parts that are smaller than $50 \%$ of the diameter of the model.

\section{Scaling}

- Scale Factor calculation: The "Measurement tool" to measure the object of known length (e.g., the scale bar included in the model) is available from the toolbar. For example, if the scale is $20 \mathrm{~cm}$ in reality, and the "Measuring" tool indicates that it is "2 $\mathrm{m}$ (MO: 2$)$ ", the scale factor is $0.2 / 2=0.1$.

- "Transform: Scale, Normalize" filter for scaling according to the scale factor, available in "Filters" > "Normals, Curvatures and Orientation". The scale factor can be entered in the "X Axis" field (the options "Freeze Matrix" and "Uniform Scaling" need to remain set), after which the scale can be applied to the model. The model may disappear from view after this step; calling "Windows" > "View from" > "Front" will centre it again.

\section{Fitting of the horizontal (xy) plane and orientation}

- Model selection: use, e.g., the "Select Faces in a rectangular region" tool, which is available from the toolbar. A rectangle has to be drawn either around the whole model or a part of it that shall serve to define the horizontal plane. The selection will then appear in red.

- Apply the "Transform: Rotate to fit to a plane" filter ("Filters" > "Normals, Curvatures and Orientation"), left at default options. Make sure the "Move to Origin" option is set so that the fitted model will be centred. After applying the filter, apply "Filters" > 
"Selection" > "Select None" to undue the selection. The fitted model can be viewed in top view (along the z-axis) by applying "Windows" -> "View from" -> "Front".

- Activate the "Manipulators Tool" (available from the toolbar) to rotate the model around the z-axis. Press the "R" key to enable rotation, the " $Z$ " key to select the z-axis, and the "Space" key to enable rotation around the centre of the model. Rotation can then be done interactively with left click + drag. To accept the changes press the "enter" (pc) or "return" (mac) key, and reject changes with the "del" (pc) or "delete" (mac) key. Before exporting the model, the matrix needs to be freezed ("Filters" > "Mesh Layer" > "Matrix: Freeze current matrix").

- If the model is flipped upside-down, it is easiest to use the "Transform: Rotate" filter ("Filters" > "Normals, Curvatures and Orientation"). In the filter dialog, rotation on " $X$ axis" or "Y axis" should be selected and an angle angle of $180^{\circ}$ specified.

\section{D VISUALISATIONS}

\section{Model import and texture activation}

- In MeshLab, a model can be opened by calling "File" > "Import Mesh...", or simply by dragging the model file into the program window. The texture, if available, will be loaded automatically. It can be disabled by setting "Texture Coord" to "Off" in the "Layer dialogue" ("View" > "Show layer dialogue"; opened by default).

- In ParaView, a model can be opened using the "File" > "Open..." dialogue (or by dragging the model file into the program window), and by pressing the "Apply" button of the "Properties" pane ("View" > "Properties"; opened by default). If needed, the orientation of the model can be corrected using the "set view direction" buttons available from the toolbar (the $-Z$ button will set the model to top view if the model was correctly oriented with MeshLab as explained above).

- The texture is not loaded automatically in ParaView. The texture file (which can be, e.g., a jpg) can be selected and loaded using the "Texture" option in the "Lighting" 
section of the "Properties" pane. This section will only be visible after toggling the "advanced properties" using the gear icon in the upper right of the "Properties" pane.

\section{File export}

- In MeshLab, the 2D visualisation currently visible on the screen can be exported using the "Save snapshot" feature (available from the toolbar). Resolution of the output file can be improved by increasing the "Screen Multiplier" setting. Before image export, distracting control elements such as the track ball and the info panel can be disabled using the "View" menu. The background may be changed/disabled with the "Background" option of the "Save snapshot" dialogue.

- In Paraview, the graphical output can be exported by selecting "File" > "Save Screenshot..." from the menu. Resolution of the output file can be adjusted in the "Image Resolution" option of the "Save Screenshot Options" dialogue; the "x" button allows to quickly double the requested resolution. Note that text and lines, including the scale bar, background grid, and contours (but not the colour legend), will not be scaled proportionally; it is therefore necessary to increase their width and size manually before screenshooting by the same factor with which the "Image Resolution" values are to be increased.

- The background may be changed/disabled using the "Override colour Palette" option of the same dialogue.

- In CloudCompare, 2D visualisations are exported by calling "Display" > "Render to File". To change the background to white, and the text and scale bar to black, go to "Display" > "Display options: colours and Materials: Colours" and select and change the "background" and "text" entries accordingly.

\section{Scale bars and background grids}

- In MeshLab, a background grid for scale can be added by using the "Render" > "Background Grid" option. After calling this option, a new entry ("Background Grid") will 
appear in the "Layer Dialogue", where the "Box Ratio" may be set to "1", and the "Major" and "Minor Spacing" (defining the size of the grid squares) adjusted as needed.

- In ParaView, a background grid can be added by checking the "Axes Grid" entry in the "Annotations" section of the "Properties" pane (available only after toggling the "advanced properties" as described above).

- In ParaView, a scale bar can be added using the "Ruler" function ("Sources" > "Measurement tools" > "Ruler"; press "Apply" in the "Properties" pane to load). In the "Properties" pane, the "Show Line" checkbox in the "Distance" section should be unchecked. Per default, the scale bar is centred on the model and $1 \mathrm{~m}$ in length (-0.5 to 0.5 ). To adjust position and length, the values for "Point1" and "Point2" ( $x, y$, and $z$, respectively) in the "Distance" section of the "Properties" pane can be changed (e.g., values of "0", "-1", "0" for "Point1" and "0.1", "-1" , "0" for "Point2" will set the scale bar to $0.1 \mathrm{~m}$ and move it $1 \mathrm{~m}$ towards the bottom of the plot). The unit can be added to the scale legend using the "Label Format" option in the "Label Properties" section by adding the desired text (e.g., " $\mathrm{m}$ ") to the end of the line (e.g., "\%6.3g m"). The "Scale" option in the "Label Properties" section can be used to change the displayed unit; e.g., setting this value to "100" changes the displayed number from $\mathrm{m}$ to $\mathrm{cm}$. Line colour can be changed using the "Axis Colour" option in the "Axis Display Properties" dialogue in the "Properties" pane, and Font properties in the "Font Properties" section. Note that settings can be set to default using the "Save current settings values as default" icon on the right of the respective higher-ranked section heading ("Properties (Ruler1)" for position and length and "Display (RulerSourceRepresentation)" for formatting, respectively).

\section{Orthographic projection}

- In MeshLab, orthographic projection can be switched on and off by calling "View" > "Toggle Orthographic Camera" from the menu. The current setting is indicated in the "FOV:" entry in the "info" panel ("View" > "Show Info Panel"; appears at the bottom of the screen). 
- In ParaView, the respective setting is termed "Camera Parallel Projection" and can be found in the "Orientation Axes" section in the "Properties" pane. Note that activation of this option will only be valid for the current session; we therefore recommend to set it as default using the "Save current view settings as default" icon on the left of the "View (Render View)" section header.

In CloudCompare, click the "Set current view mode" icon (available in the left vertical toolbar), and select "Orthographic projection".

\section{Colour information}

- Vertex colours can be corrected in MeshLab for brightness and contrast using "Filters" $>$ "Colour creation and processing" > "Vertex colour brightness contrast gamma".

\section{Orthophoto generation}

- In MeshLab, the equivalent output can be generated by setting to "orthographic projection", enabling the texture, disabling vertex colours (setting "Colour" to "Mesh"), and disabling shading (setting "Shading" to "None"; all options available in the "Layer Dialog" pane).

- In ParaView, the texture has to be loaded, camera parallel projection enabled, and the model oriented in top view (see instructions above). To generate a pure orthophoto without oblique light shading, the "Light Kit" can be disabled in the "Light Inspector" pane (which can be activated in the "View" menu).

\section{Height maps (ParaView)}

- To produce a basic map, call the "Elevation" filter ("Filters" > "Alphabetical"). Note that the filter will only be available when the model is selected in the "Pipeline Browser". After calling the filter, the $z$-axis need to be selected as reference by pressing the " $Z$ Axis" button in the "Properties" pane, and the map generated by pressing the "Apply" 
button. The line showing the direction of the filter should be hidden by unchecking the "Show Line" in the "Properties" pane.

- Different colour scales can be selected by pressing the "Choose preset" button within the "Mapping Data" section of the "Colour Map Editor" pane. The latter can be activated by checking "View" > "Colour Map Editor". To fill the lowest and/or highest parts of the model with uniform colour, the "rescale to custom range" option of the "Colour Map Editor" (second button of the vertical right-hand button bar in the "Mapping Data" section) may be used. For example, values of 0.2 and 0.8 will condense the colour scale to the middle $60 \%$ of the total height of the model. The colour distribution can furthermore be changed by adjusting the control points of the "Colour transfer function editor" of the colour Map Editor (horizontal colour bar in the "Mapping Data" section) to improve the visibility of details of interest. A colour scale can be inverted by pressing the "Invert the transfer functions" button in the "Mapping Data" section.

- The colour legend will be shown by default, but can be toggled by pressing the "Toggle colour Legend Visibility" button available in the toolbar. Per default, the legend will only display relative values from 0 to 1 (corresponding to the lowest and highest elevations, respectively) rather than the actual total depth of the model in metres. The absolute model depth is indicated by the " $Z$ range" delta value as seen in the "Bounds" section of the "Information" pane ("View" > "Information"). To change to an absolute scale in metre, this value can be copied to the second field of the "Scalar Range" option in the "Properties" Panel (available only when the "Elevation1" entry in the "Pipeline Browser" is activated). The plot and colour legend can then be updated by clicking "Apply" in the "Properties" pane, and clicking "Rescale to data range" (first button of the vertical toolbar in the "Mapping Data" section of the "Colour Map Editor"). The decimal place of the value entered in the "Scalar Range" field can be changed to achieve a scale in centimetres or millimetres instead of metres.

- The lowest and highest values on the colour scale may be displayed in real number format by entering, "\%-\#6.2f" into the "Range Label Format" in the "Labels" section of the "Edit colour Legend Properties" (icon in the upper right corner of the "Colour Map 
Editor"). The option is available after toggling the advanced properties (gear icon in the upper right of the same dialogue). The lowest and highest values can alternatively disabled by unchecking "Add Range Labels" in the same section.

- If the image is to be exported with a white background, the fonts of the colour legend can be changed from the default white to black. This is done using the "Edit colour Legend Properties" dialogue, which is available after clicking the icon of the same name in the upper right corner of the "Colour Map Editor". In the dialogue, both the "Title Font Properties" and the "Text/Annotation Font Properties" need to be changed.

- The elevation, direction, intensity and warmth of the light source responsible for the shading can be changed using the "Light Inspector" pane ("View" > "Light Inspector"). See section "Shaded reliefs" for more details. Disabling of the Light Kit disables any shading.

\section{Height maps (CloudCompare)}

- In CloudCompare, height maps can be generated by adding a scalar field in Z-direction ("Edit" > "Scalar fields" > "Export coordinates to SF"; available when the mesh is selected). The colour map can then be activated by selecting "Toggle SF" from the context menu of the "Mesh" entry of the "DB Tree" pane.

- Various colour schemes are available in the "Colour Scale" section of the "Properties" pane (visible when the model is selected in the "DB Tree" pane), and can be edited using the "Colour Scale Editor" (the gear icon in the "Colour Scale" section). The default scheme, the four-colour "Blue>Green>Yellow>Red", works well for most ichnological objects, as it provides sufficient, but not excessive, colour diversity. As in ParaView, the colour range can be edited to fill very high or low parts of the model that are not of interest with uniform colour; this can be achieved by changing the "saturation" values in the "SF display params" section of the "Properties" pane.

- A colour legend can be added by checking the "Visible" checkbox in the "Colour Scale" section of the "Properties" pane. In contrast to ParaView, the colour legend will display absolute rather than relative values. 
- The position of the light source can be changed interactively by simultaneously pressing Ctrl and the right mouse button.

\section{Contour plots (ParaView)}

- Call the "Contour" function ("Filters" > "Common"), which is available after application of the "Elevation" function and selection of the "Elevation1" entry in the "Pipeline Browser".

- Make sure that "Contour1" is selected in the "Pipeline Browser". Remove pre-defined contours by clicking the "Remove all entries" icon in the "Isosurfaces" section of the "Properties" pane.

- Enter the desired number of contours in the "Number of Samples" field of the "Generate Number Series" dialogue, which is available after clicking the "Add a range of values" button in the "Isosurfaces" section of the "Properties" pane.

- For relative contours: Note that the first and last entry in the value range (the minimum and maximum height, respectively) will not be plotted; 7 steps will therefore result in 5 contours displayed.

- Absolute contours are available after the "Scalar Range" of the "Properties" pane of the "Elevation" filter was set according to the total height of the model, as explained above under "Height maps (ParaView)". For absolute contours, the left field in the "Range" section in the "Generate Number Series" dialogue should be set to the desired distance between contours, and the value displayed in the right field should be rounded down to a next multiple of that distance. The required number of contours needs to be calculated (value of the left field/value of the right field) and entered in the "Number of Samples" field. For example, if the total height of the model is $0.068 \mathrm{~m}$, and the desired distance between contours is $0.01 \mathrm{~m}$, then the left field should be " 0.01 ", the right field "0.06", and the "Number of Samples" field "6".

- The contours can then be generated by clicking "Apply" in the "Properties" pane. Per default, the contours are coloured according to the colour scale selected in the "Elevation" filter. To change to a solid colour, select "Solid colour" in the first drop-down 
menu of the "Colouring" section of the "Properties" pane. The desired colour can then be picked by clicking the "Edit" field in that same section. Best results when exporting at high resolution can be achieved by selecting "Points" in the "Representation" dropdown menu, which can be found in the section "Point Locator" in the "Properties" pane. Thickness of contours can be changed using the "Line Width" (when "Representation" is set to "Surface") and "Point Size" (when "Representation" is set to "Points"); both are available in the "Styling" section of the "Properties" pane with advanced options enabled.

- A contour plot may be combined with other layers of the "Pipeline browser" (e.g., a height map) by setting the respective entries in the "Pipeline browser" to visible. It is possible to combine two types of contours in the same plot (e.g., formatting each fifth contour with a greater width and different colour) by applying a second contour plot.

\section{Contour plots (CloudCompare)}

- The "Rasterize" tool of CloudCompare does not work on meshes but on point clouds; therefore, the vertex points of the model need to be selected as input (the "Vertices" entry in the "DB Tree", which is hidden by default and appears when clicking on the arrow before the "Mesh" entry). Now, the tool can be started from the menu ("Tools" > "Projection"). In the "Rasterize" dialogue, the "Fill with" option of the "Empty cells" section should be set to "interpolate", and the grid generated by pressing the "Update grid" button.

- After the grid was created, absolute contours can be created using the "Contour plot" tap of the "Rasterize" dialogue. Four parameters may be changed: The "Start value" defines the level of the first contour (in metres; this value may be set to "0"). The "Step" value defines the distance between contours (in metres). The "Min. vertex count" allows for filtering very short contours (e.g., with a value of 50, all contours of less than 50 vertex points will not be displayed). The "Line width" specifies the width of the individual contours, and frequently needs to be increased especially if images of higher resolution need to be exported. If the "Colourize" option is checked, the separate contours are coloured according to their height using the active colour scale. The 
contour plot is generated, and a preview displayed, after pressing the "Generate" button. If correct, the contours need to be exported by pressing the "Export" button. The exported contours will be stored within a new entry in the "DB Tree", and can be combined with other entries, such as height maps.

- The contour colour can be changed (e.g., to "black") by calling "Display" > "Display options" and, in the "Colours and Materials" section, changing the colour of the "Points" entry.

\section{Shaded reliefs}

- In MeshLab's "Layer dialog", activate the tab for solid model rendering (the blue cylinder icon). In this tab, "Shading" can be set to "Face"; "Colour" set to "Mesh"; "Back-Face" set to "Single"; and "Texture Coord" to "off". Setting "Shading" to "Vert" instead of "Face" is possible as well and will lead to a more smoothened surface with more indistinct polygon boundaries; if any black spots are visible on the mesh surface, these can be resolved by applying "Filters" > "Cleaning and Repairing" > "Snap Mismatched Borders". The position of the light source can be changed interactively by pressing Ctrl+Shift and dragging with the mouse over the model.

- In ParaView, the position of the light source has to be entered using the "Light Inspector" pane (to be activated by checking "View" > "Light Inspector"). If a texture or height map was already applied, they should be disabled beforehand (setting "Texture" to "None" in the "Properties" pane, and deactivating the Elevation entry in the "Pipeline Browser", respectively). Also, colouring should be changed to "Solid colour" in the "Colouring" section of the "Properties" pane. Within the "Light Inspector", the default "Light Kit" should be disabled, and a new light source added ("Add Light"). Important parameters are the "Intensity" and "Light Position" values. For example, an intensity of "2" and a position of "-1", "1", and "0.2" (along the $x, y$, and z-axis, respectively) will produce low-angled light from the upper left and works well with surfaces of relatively low relief. For surfaces with higher relief, the $z$ value (the height of the light source) can be increased. 
- In ParaView, the position of both the light source and focal point can be changed interactively. After following the steps described above, a white line can be seen extending from the centre of the model, provided that the model was centred on the origin of the coordinate system, as described above. This line connects the focal point (at the centre of the model) to the light source (a white circle; zoom out if not seen). The light source can be dragged around the model with the mouse; decreasing its distance to the model will increase intensity.

- In CloudCompare, the light source can be changed interactively by pressing Ctrl+right click.

\section{Surface inclination plots (ParaView)}

- To produce a surface inclination plot, the vertex normals have to be stored within the model file. This can be achieved when exporting the model in MeshLab by activating the "Normal" option in the "Choose Saving Options" dialogue that will appear when attempting to save a model in MeshLab. Furthermore, it may be necessary to apply "Filters" > "Cleaning and Repairing" > "Snap Mismatched Borders" in MeshLab to avoid artefacts in the form of black spots on the image output.

- Select "Normals" in the first drop-down menu of the "Colouring" section of the "Properties" pane. Select " $Z$ " in the second drop-down menu. In the "Colour Map Editor", select the "X Ray" preset using the "Choose preset" button, and invert the colour scale using the "Invert the transfer functions" button. Restrict the range of the colour map using the "Rescale to custom range" button. Models with very low relief require a more severe restriction (e.g., 0.95-1) than models with higher relief (e.g., 0.6-1). For a pure inclination plot without additional shading, disable the "Light Kit" using the "Light Inspector" pane.

- For directional plots equivalent to a shaded relief, choose " $\mathrm{X}$ " or "Y" instead of " $Z$ " in the "Colouring" section. Restrict the colour scale (e.g., -0.2-1) to reach the desired output. 


\section{Ambient occlusion plots (MeshLab)}

- Apply the "Ambient occlusion" filter (available in "Filters" > "Colour Creation and Processing"), and, in case the model includes a texture, set "Texture Coord" to "Off" in the "Layer dialogue".

- Ambient occlusion plots calculated by MeshLab tend to be overexposed. This can be corrected using the "Vertex colour Level Adjustment" filter (available in "Filters" > "Colour Creation and Processing"), where the "Gamma" and "Max output level" values need to be lowered appropriately (use the "Preview" option provided by the filter for a live preview).

\section{Ambient occlusion plots (CloudCompare)}

- Apply the Ambient Occlusion filter via either the "P.C.V." icon from the top menu bar or from "Plugins" > "P.C.V. (Ambient Occlusion)", using the default "ShadeVis" settings. In the Properties menu panel, uncheck "Materials/textures".

- Ambient occlusion tend to be overexposed to a lesser degree when compared with Meshlab. After saving the image in CloudCompare ("Display" > "Render to file"), the overexposure be corrected in image editors (e.g., GIMP) by adjusting the "Levels" or "Brightness/Contrast".

\section{Radiance Scaling (Gratin)}

- In Gratin, open the pipeline file "radiance-scaling-gratin-pipeline.gra" provided in the supplementary information of the present paper using "File" > "Open". The pipeline of nodes will appear in the lower pane of the program; zoom in and out with the mouse wheel and right click + drag with the mouse to scroll.

- To load an OBJ model, double-click into the leftmost node of the pipeline ("genericObjLoader"), click "Load" in the "Node parameters" pane to select the model, and press "Reset cam". Three graphical outputs applying radiance scaling with different settings can be found on the far right of the pipeline (labeled 
"enhancedShading"). The outputs can be displayed in the "View/Interact" pane by double-clicking into them and pressing the "space" key.

- The strength of the filter can be changed using the "gamma" parameter in the "Node parameters" of the respective node. The size of the features that are to be enhanced is determined by the "sigmaS" parameter, which can be changed in the respective previous node (labeled "bilateral Filter") after double-clicking into them. The resolution of the image to be export can be increased in the Settings of the "genericObjLoader" node (increase "W+" and "H+").

- The image that is loaded into the "View/Interact" pane can then be exported with "Tools" > "Export node output".

\section{Cross sections (CloudCompare)}

- The point cloud of the mesh needs to be selected (the "Vertices" entry in the "DB Tree", available after clicking on the black arrow), and all three levels in the DB Tree including the Mesh and Vertices activated. Then, the segmentation feature is to be called ("Tools" > "Segmentation" > "Extract Sections / Unfold"), which allows to draw the line by mouse clicking (a left click will add section points, a right click will finish the defined line). After the line was defined, the "polyline edition" mode needs to be disabled by clicking the "Create polyline by clicking on the screen" tool (the first entry in the toolbar that appeared on the upper right of the model window). The section can now be exported using the "Extract points along active sections" tool of the same toolbar. The extracted profile is automatically added to the "DB Tree", and the tool can be closed using the green tick icon.

- Before data export, the profile needs to be rotated so that the view is exactly vertical to the line. This rotation can be applied to the "Section contour" (which needs to be selected in the DB Tree) using the "Fit Plane" and "Apply transformation" functions.

After rotation, the line can be centred on the display by selecting it in the "DB Tree" and pressing $Z$. 
- Coordinates of the cross section line can be exported by selecting the "vertices" within the "Extracted profiles" entry of the DB Tree, followed by applying "File" > "Save".

\section{Measurements on 2D visualisations (Inkscape)}

- Before measuring, the measurement tool should be set to centimetres and the scale factor in percent specified in the "Scale \%" box (the value can be calculated by measuring a scale on the image, ideally the "Axes Grid" drawn by ParaView).

- Measurements can then be done with the "Measurement" tool, available from the toolbar or by pressing the "M" key. Measurements are done by left-clicking and dragging.

- Measurements can be saved to the document for future reference using the "Mark Dimension" function, preferably on a new layer.

- Angles can be measured by left click and drag, which measures an arbitrary line against a horizontal line; the latter can be changed by hitting the shift key.

\section{Tracings and image stacks (Inkscape)}

- Tracings can be done point-by-point using the "Draw Bezier curves and straight lines" tool. Smoothing of the completed tracing can be done by selecting the path, pressing F2 to allow for node editing, selecting all nodes of the path, and applying the "Make selected nodes smooth" function available in the main toolbar.

- Alternatively, tracings can be done by dragging the mouse using the "Draw freehand lines" tool. Increase the "Smoothing" parameter in the main toolbar to create less jagged tracings.

- A precise overlay of multiple visualisations can be most easily produced when all images have been exported using the same application, such as ParaView. 
- To produce the image stack, the imported images should be scaled proportionally to the same width (or height) using the "Transform" function, and centred on both the horizontal and vertical axis using the "Align and Distribute" tool.

\section{Trackway calculator (Inkscape and LibreOffice Calc)}

- To allow for coordinate export in Inkscape, the "Export xy" plugin (provided in the supplementary information) needs to be installed. The two files "ExportXY.inx" and "ExportXY.py" need to be placed into the folder "extensions" within the Inkscape program directory, and Inkscape restarted. The plugin will then be available by calling "Extensions" -> "Export" -> "Export xy" from the Inkscape menu bar.

- Before coordinate export, it has to be ascertained that the document scale is set to "1" (the "Scale x:" field in the "Page" pane of the "Document Properties", which is available from the "File" menu).

- Coordinates are placed in Inkscape using the "Draw Bezier curves and straight lines" tool. After the "Z" coordinate has been placed on the first footprint (e.g., LP1, the first left pes impression), the "L1" coordinate of the next footprint (e.g., LM1, the first left manus impression) is placed, and so on, resulting in a connected sequence of straight lines capturing the whole trackway.

- To improve the accuracy in fitting the footprint axes, centres, and perpendicular lines, grid templates may be placed over each footprint prior drawing of the lines. These templates, which are provided in the example Inkscape file in the supplementary information, can be easily scaled and rotated to fit the respective footprint.

- If one or several coordinates cannot be placed due to incomplete or missing footprints, placeholder coordinates have to be placed to the right of the trackway figure of the sheet (e.g., an entirely missing footprint requires five placeholder coordinates). These placeholder coordinates need to have a $\mathrm{x}$ coordinate greater than a number to be specified in the "Trackway calculator" form ("Ignore coords with $x$ greater as:").

- The output of the "ExportXY" plugin may than be copied and pasted into the third and forth columns of the first sheet of the "Trackway calculator". 
- After all data is entered, the document should be recalculated by pressing "F9". 